\title{
THE BRIGHTEST YOUNG STAR CLUSTERS IN NGC 5253*
}

D. Calzetti $^{1}$, K. E. Johnson ${ }^{2}$, A. Adamo ${ }^{3}$, J. S. Gallagher III ${ }^{4}$, J. E. Andrews ${ }^{5}$, L. J. Smith $^{6}$, G. C. Clayton ${ }^{7}$, J. C. LeE $^{8,9}$,

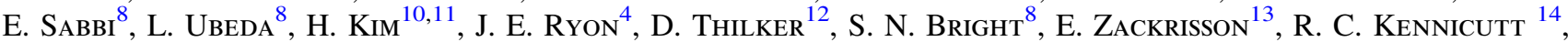
S. E. De Mink ${ }^{15}$, B. C. Whitmore ${ }^{8}$, A. Aloisi ${ }^{8}$, R. Chandar $^{16}$, M. Cignoni $^{8}$, D. CoOK $^{17}$, D. A. Dale ${ }^{17}$, B. G. ElmeGreenN ${ }^{18}$, D. M. Elmegreen ${ }^{19}$, A. S. Evans $^{2,20}$, M. Fumagalli $^{21}$, D. A. Gouliermis ${ }^{22,23}$, K. Grasha ${ }^{1}$, E. K. Grebel ${ }^{24}$,

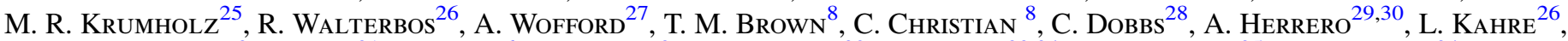
M. Messa ${ }^{3}$, P. Nair ${ }^{31}$, A. Nota ${ }^{6}$, G. Östlin ${ }^{3}$, A. Pellerin ${ }^{32}$, E. SACChi ${ }^{33,34}$, D. Schaerer ${ }^{35}$, and M. Tosi ${ }^{34}$

${ }^{1}$ Department of Astronomy, University of Massachusetts-Amherst, Amherst, MA 01003, USA; calzetti@astro.umass.edu

${ }^{2}$ Department of Astronomy, University of Virginia, Charlottesville, VA, USA

${ }^{3}$ Department of Astronomy, The Oskar Klein Centre, Stockholm University, Stockholm, Sweden

${ }^{4}$ Department of Astronomy, University of Wisconsin-Madison, Madison, WI, USA

${ }^{5}$ Department of Astronomy, University of Arizona, Tucson, AZ, USA

${ }^{6}$ European Space Agency/Space Telescope Science Institute, Baltimore, MD, USA

${ }^{7}$ Department of Physics and Astronomy, Louisiana State University, Baton Rouge, LA, USA Space Telescope Science Institute, Baltimore, MD, USA

${ }^{9}$ Spitzer Science Center, Caltech. Pasadena, CA, USA

${ }^{10}$ Department of Astronomy, The University of Texas at Austin, Austin, TX, USA

${ }^{11}$ Korea Astronomy and Space Science Institute, Daejeon, Korea
${ }^{11}$ Department of Physics and Astronomy, The Johns Hopkins University, Baltimore, MD, USA

${ }^{13}$ Department of Physics and Astronomy, Uppsala University, Uppsala, Sweden

${ }^{14}$ Institute of Astronomy, University of Cambridge, Cambridge, UK

15 Anton Pannekoek Institute for Astronomy, University of Amsterdam, Amsterdam, The Netherlands

${ }^{16}$ Department of Physics and Astronomy, University of Toledo, Toledo, OH, USA

17 Department of Physics and Astronomy, University of Wyoming, Laramie, WY, USA

18 IBM Research Division, T.J. Watson Research Center, Yorktown Hts., NY, USA

19 Department of Physics and Astronomy, Vassar College, Poughkeepsie, NY, USA

${ }^{20}$ National Radio Astronomy Observatory, Charlottesville, VA, USA

${ }^{21}$ Institute for Computational Cosmology and Centre for Extragalactic Astronomy, Department of Physics, Durham University, Durham, UK

${ }^{22}$ Centre for Astronomy, Institute for Theoretical Astrophysics, University of Heidelberg, Heidelberg, Germany

24 Max Planck Institute for Astronomy, Heidelberg, Germany

Astronomisches Rechen-Institut, Zentrum für Astronomie der Universität Heidelberg, Heidelberg, Germany

${ }^{25}$ Department of Astronomy \& Astrophysics, University of California-Santa Cruz, Santa Cruz, CA, USA

${ }^{26}$ Department of Astronomy, New Mexico State University, Las Cruces, NM, USA

${ }^{27}$ UPMC-CNRS, UMR7095, Institut d'Astrophysique de Paris, Paris, France

${ }^{28}$ School of Physics and Astronomy, University of Exeter, Exeter, UK

${ }^{29}$ Instituto de Astrofisica de Canarias, La Laguna, Tenerife, Spain

${ }^{30}$ Departamento de Astrofisica, Universidad de La Laguna, Tenerife, Spain

31 Department of Physics and Astronomy, University of Alabama, Tuscaloosa, AL, USA

${ }^{32}$ Department of Physics and Astronomy, State University of New York at Geneseo, Geneseo, NY, USA

33 Dipartimento di Fisica e Astronomia, Università degli Studi di Bologna, Bologna, Italy

${ }^{34}$ INAF-Osservatorio Astronomico di Bologna, Bologna, Italy

35 Observatoire de Genève, University of Geneva, Geneva, Switzerland

Received 2015 June 8; accepted 2015 August 17; published 2015 September 24

\begin{abstract}
The nearby dwarf starburst galaxy NGC 5253 hosts a number of young, massive star clusters, the two youngest of which are centrally concentrated and surrounded by thermal radio emission (the "radio nebula"). To investigate the role of these clusters in the starburst energetics, we combine new and archival Hubble Space Telescope images of NGC 5253 with wavelength coverage from $1500 \AA$ to $1.9 \mu \mathrm{m}$ in 13 filters. These include $\mathrm{H} \alpha, \mathrm{P} \beta$, and $\mathrm{P} \alpha$, and the imaging from the Hubble Treasury Program LEGUS (Legacy Extragalactic UV Survey). The extraordinarily wellsampled spectral energy distributions enable modeling with unprecedented accuracy the ages, masses, and extinctions of the nine optically brightest clusters $\left(M_{V}<-8.8\right)$ and the two young radio nebula clusters. The clusters have ages $\sim 1-15$ Myr and masses $\sim 1 \times 10^{4}-2.5 \times 10^{5} M_{\odot}$. The clusters' spatial location and ages indicate that star formation has become more concentrated toward the radio nebula over the last $\sim 15 \mathrm{Myr}$. The most massive cluster is in the radio nebula; with a mass $\sim 2.5 \times 10^{5} M_{\odot}$ and an age $\sim 1 \mathrm{Myr}$, it is $2-4$ times less massive and younger than previously estimated. It is within a dust cloud with $A_{V} \sim 50$ mag, and shows a clear near-IR excess, likely from hot dust. The second radio nebula cluster is also $\sim 1$ Myr old, confirming the extreme youth of the starburst region. These two clusters account for about half of the ionizing photon rate in the radio nebula, and will eventually supply about $2 / 3$ of the mechanical energy in present-day shocks. Additional sources are required to supply the remaining ionizing radiation, and may include very massive stars.
\end{abstract}

\footnotetext{
* Based on observations obtained with the NASA/ESA Hubble Space Telescope, at the Space Telescope Science Institute, which is operated by the Association of Universities for Research in Astronomy, Inc., under NASA contract NAS 5-26555.
} 
Key words: galaxies: dwarf - galaxies: general - galaxies: individual (NGC 5253) - galaxies: starburst galaxies: star clusters: general

\section{INTRODUCTION}

Local dwarf starburst galaxies are close counterparts to the high-redshift star-forming systems that built today's galaxies via interactions and mergers. The investigation of nearby dwarfs that are undergoing starburst events may, thus, shed light on the way galaxies assemble their stellar populations across cosmic times, and on the role young massive star clusters have in the energy and mechanical output of star formation.

The extreme youth of the starburst in the center of the dwarf galaxy NGC 5253 has been established by many investigators (e.g., van den Bergh 1980; Moorwood \& Glass 1982; Rieke et al. 1988; Caldwell \& Phillips 1989; Beck et al. 1996; Calzetti et al. 1997; Pellerin \& Robert 2007), and continues to be supported by recent data. The majority of the star clusters located within the central $\sim 250-300 \mathrm{pc}$ has ages in the range from $\sim 10^{6}$ years to a few $10^{7}$ years (Calzetti et al. 1997; Tremonti et al. 2001; Harris et al. 2004; Chandar et al. 2005; Cresci et al. 2005; de Grijs et al. 2013). A few older clusters, up to $\sim 10^{10}$ years in age, are located farther away from the galaxy's center (Harbeck et al. 2012; de Grijs et al. 2013). The youth of the central starburst is further supported by the absence of detectable non-thermal radio emission (Beck et al. 1996) and the presence of strong signatures from WolfRayet stars (Campbell et al. 1986; Kobulnicky et al. 1997; Schaerer et al. 1997; Lopez-Sanchez et al. 2007; Monreal-Ibero et al. 2010; Westmoquette et al. 2013), which set a limit of $\lesssim 3-4$ Myr to the most recent episode of star formation. The age range of the diffuse UV stellar population (Tremonti et al. 2001; Chandar et al. 2005) and the recent star formation history of NGC 5253 (McQuinn et al. 2010; Harbeck et al. 2012) indicate that the star formation has been elevated, relative to the mean Hubble time value, for the past $\sim 5 \times 10^{8}$ years.

The question of how to sustain continuously elevated star formation, possibly in the form of subsequent bursts, in NGC 5253 has been tackled by many authors. An encounter with the relatively nearby grand-design spiral M83 about $1 \mathrm{Gyr}$ ago has been suggested as one of the potential initial triggers (e.g., van den Bergh 1980; Caldwell \& Phillips 1989). M83 is located at a distance of $4.5 \mathrm{Mpc}$ (Thim et al. 2003) and is $1^{\circ} 54^{\prime}$ to the NW of NGC 5253; thus, M83 is separated from NGC 5253 (at a distance of $3.15 \mathrm{Mpc}$ Freedman et al. 2001; Davidge 2007) by about $1.35 \mathrm{Mpc}$. Although the distance is significant, and although Karachentsev et al. (2007) place NGC 5253 in the neighboring Cen A subgroup, Lopez-Sanchez et al. (2012) argue that NGC 5253 is located at the boundary between the two subgroups of Cen A and M83. ${ }^{36}$ A past interaction with the latter galaxy could explain the tidal extension in $\mathrm{HI}$ to the SE of M83 and the extension to the North of the HI distribution in NGC 5253. These tails could be providing the fuel for the past and current bursts of star formation in NGC 5253, in the form of in-falling metal-poor HI

\footnotetext{
36 There are still uncertainties in the actual distances of both M83 and NGC 5253. Karachentsev et al. (2007) place M83 at a distance of 5.2 Mpc, and Sakai et al. (2004) place NGC 5253 at a distance of $3.6 \mathrm{Mpc}$, the latter much closer to the distance of Cen A, 3.8 Mpc. This has led Karachentsev et al. (2007) to associate NGC 5253 to the Cen A subgroup. In the latter case, NGC 5253 may have interacted with Cen A, instead of M83, in the past.
}

clouds (Lopez-Sanchez et al. 2012). The in-falling clouds convert to higher density molecular gas once they enter the central galaxy region and mix with the local interstellar medium (ISM; Turner et al. 1997, 2015; Meier et al. 2002). The potential entrance "channel" for the gas is defined by the only prominent dust lane, which bisects the galaxy roughly along the minor axis and emits in CO (Walsh \& Roy 1989; Meier et al. 2002; Turner et al. 2015).

Thus, the current starburst in NGC 5253 is possibly the latest episode of a series of such feeding events, which are still ongoing. The dust-corrected UV and $\mathrm{H} \alpha$ luminosities both provide a consistent value of the star formation rate, $\mathrm{SFR}=0.1-0.13 M_{\odot} \mathrm{yr}^{-1}$ (Calzetti et al. 2004, 2015), also in agreement with the SFR derived from the total infrared emission, $\mathrm{SFR}(\mathrm{TIR})=0.1 M_{\odot} \mathrm{yr}^{-1}$ (using $L_{\mathrm{TIR}}=$ $3.7 \times 10^{42} \mathrm{erg} \mathrm{s}^{-1}$, which we calculate from the Spitzer imaging data of Dale et al. 2009). Radio measurements at $0.3,0.7,1.3$, and $2 \mathrm{~cm}$ of the free-free emission (Turner et al. 2000; Meier et al. 2002; Turner \& Beck 2004) yield a SFR $\sim 0.3-0.36 M_{\odot} \mathrm{yr}^{-1}$ which is roughly a factor of three higher than what is obtained from the TIR and from the dustattenuation-corrected $\mathrm{UV}$ and $\mathrm{H} \alpha$. The relatively small $\mathrm{H} \alpha$ and UV half-light radii, $\sim 100 \mathrm{pc}$ and $\sim 160 \mathrm{pc}$, respectively (Calzetti et al. 2004), imply a high star formation rate density, $\Sigma_{\mathrm{SFR}} \sim 3.5 M_{\odot} \mathrm{yr}^{-1} \mathrm{kpc}^{-2}$, confirming the star bursting nature of the galaxy (Kennicutt \& Evans 2012). The specific SFR of NGC 5253 is sSRF $\sim 0.6-1.4 \times 10^{-9} \mathrm{yr}^{-1}$, for a stellar mass $M_{*} \simeq 2.2 \times 10^{8} M_{\odot}($ Calzetti et al. 2015); the galaxy lies above the Main Sequence of star formation, i.e., the SFR versus stellar mass relation, for local galaxies (Cook et al. 2014), as expected for a starburst.

Most of the current activity is coincident with a centrally concentrated, dusty radio source about $15-20 \mathrm{pc}$ in extent, which we term the "radio nebula." This has enough free-free emission to require one or more $\lesssim 3$ Myr old star clusters with total mass $M \sim 10^{6} M_{\odot}$ (Turner et al. 2000; Turner \& Beck 2004), for a 0.1-120 $M_{\odot}$ Kroupa stellar Initial Mass Function (IMF Leitherer et al. 1999; Kroupa 2001). The ratio of the stellar mass to gas mass in the region suggests a star formation efficiency around $60 \%$, or about 10 times higher than that of Milky Way clouds (Turner et al. 2015). At least two distinct young star clusters are identifiable in the region, one of which is heavily attenuated by dust, and has been associated with the peak of emission at 1.3, and $2 \mathrm{~cm}$ by Alonso-Herrero et al. (2004); this source has angular size 0 "'05 $\times 0$ 0!" 1 $\left(\sim 0.8 \times 1.6 \mathrm{pc}^{2}\right)$ and is associated with $\sim 20 \%-30 \%$ of the ionizing photons in the radio nebula (Turner \& Beck 2004). The other cluster is also affected by the dust contained in the radio nebula, but to a much smaller degree; it is relatively bright in the UV, and it corresponds to the peak of observed $\mathrm{H} \alpha$ emission in the galaxy (Calzetti et al. 1997). AlonsoHerrero et al. (2004) associate this UV-bright cluster with the secondary peak of emission at $1.3 \mathrm{~cm}$ (Turner \& Beck 2004). The radio nebula is driving most of the ionization in the galaxy, and the past and on-going starburst has been stirring the surrounding ISM, both chemically and energetically.

NGC 5253 is one of the few known cases containing regions of well-detected nitrogen enhancement, likely due to localized pollution from Wolf-Rayet stars in the area of the radio nebula 
(Walsh \& Roy 1989; Kobulnicky et al. 1997; Schaerer et al. 1997; Monreal-Ibero et al. 2010, 2012; Westmoquette et al. 2013). However, no other chemical "anomalies" have been convincingly detected. Tentative reports of He enhancement (Campbell et al. 1986; Lopez-Sanchez et al. 2007), also a potential sign of pollution from Wolf-Rayet or other very massive stars (VMSs), have been recently cast into doubt (Monreal-Ibero et al. 2013). The galactocentric profile of the oxygen abundance is fairly flat (Westmoquette et al. 2013), with a mean value of $12+\log (\mathrm{O} / \mathrm{H})=8.25$ (Monreal-Ibero et al. 2012), or about $35 \%$ solar, ${ }^{37}$ and with some scatter depending on assumptions for the electron temperature zone model (Westmoquette et al. 2013). This value of the oxygen abundance is similar to the one reported by Bresolin (2011), 12 $+\log (\mathrm{O} / \mathrm{H})=8.20 \pm 0.03$.

The ionized gas emission shows evidence of feedback from previous activity in the galaxy: filaments, shells, and arches characterize the $\mathrm{H} \alpha$ distribution (Marlowe et al. 1995; Martin 1998; Calzetti et al. 1999), closely followed by the X-ray emission tracing the hot gas (Strickland \& Stevens 1999; Summers et al. 2004). The $\mathrm{H} \alpha$ is mostly photo-ionized but also includes a non-negligible fraction, up to $15 \%$ in luminosity, of shock-ionization (Calzetti et al. 1999, 2004; Hong et al. 2013); the $\sim$ kpc-size shells expand at a velocity of $\sim 35 \mathrm{~km} \mathrm{~s}^{-1}$ (Marlowe et al. 1995) and have ages around 10-15 Myr (Martin 1998). Thus, the clusters and stars located in the starburst have a major impact on a number of observable characteristics of this galaxy, which would otherwise appear to be a rather unremarkable early-type dwarf.

Despite ample evidence for mechanical feedback, we will assume in this work that only a small fraction of ionizing photons escapes the galaxy. This is true for local starburst galaxies in general, where escaping fractions are less than $3 \%$ (e.g., Grimes et al. 2009; Leitet et al. 2013). Recently, Zastrow et al. (2013) have suggested that these fractions may be lower limits due to the presence, in several starburst galaxies, of optically thin ionization cones, which may act as channels for the escape of ionizing photons. These will remain mostly undetected due to the random orientation of the cones relative to the line of sight. In NGC 5253, the putative ionization cone is coincident with the dust lane (Zastrow et al. 2011) and with optically thick $\operatorname{CO}(3-2)$ emission (Turner et al. 2015). Thus, while the properties of this feature are consistent with photoionization by escaping radiation, the ionization cone may be dusty and optically thick. Furthermore, the high ionization levels that mark this feature in NGC 5253 are found in only one direction, so the solid angle of the escape zone is likely to be small (Zastrow et al. 2013). In what follows, we assume negligible escape of ionizing photons from NGC 5253, although the issue remains open.

The new high-spatial resolution UV observations presented here provide an essential wavelength for probing the massive star population and the impact of dust extinction in the radio nebula. Our goal is to quantify the properties of the star clusters in the radio nebula, in order to better understand their energetics and role within the NGC 5253 starburst. To this end, we study the stellar population content of the two star clusters using SED-modeling techniques on UV-optical-nearIR HST photometry. The photometric stellar continuum bands are supplemented with measurements of the emission lines in

\footnotetext{
37 We adopt $12+\log (\mathrm{O} / \mathrm{H})_{\odot}=8.69$, for the solar oxygen abundance value (Asplund et al. 2009).
}

the light of $\mathrm{H} \alpha, \mathrm{P} \beta$, and $\mathrm{P} \alpha$, also from $H S T$ imaging, which help to further constrain the ages and masses of the star clusters. The robustness of the SED modeling is first tested against other bright stellar clusters within the starburst region of NGC 5253, which are less affected by dust attenuation than the clusters within the radio nebula, and can, thus, provide a handle on potential degeneracies in the results for the latter.

This paper is organized as follows: Section 2 describes the observations and the archival HST data used in this investigation; Section 3 presents the cluster selection and photometry; Section 4 presents the synthetic photometry and the fitting approach to the observed one; Section 5 describes the results of the SED fitting, and provides the ages, masses, and extinctions of the clusters, which are further discussed in Section 6. A summary and the conclusions are provided in Section 7.

\section{OBSERVATIONS AND ARCHIVAL DATA}

\subsection{New Observations}

NGC 5253 was observed with the HST Wide Field Camera 3 (WFC3) in the UVIS channel, in the filters F275W and F336W, on 2013 August 28, as part of the HST Treasury program LEGUS (Legacy ExtraGalactic UV Survey, GO-13364). A description of the survey, the observations, and the image processing is given in Calzetti et al. (2015).

Briefly, the WFC3/UVIS datasets were processed through the CALWF3 pipeline version 3.1.2 once all the relevant calibration files (bias and dark frames) for the date of observation were available in MAST. The calibrated, flatfielded individual exposures were corrected for charge transfer efficiency losses by using a publicly available stand-alone program. ${ }^{38}$ These corrections were small because we used the post-flash facility ${ }^{39}$ to increase the background to a level near $12 \mathrm{e}^{-}$. The processed individual dithered images were then aligned, cosmic-ray cleaned, sky-subtracted, and combined at the native pixel scale using the ASTRODRIZZLE routine, ${ }^{40}$ to an accuracy of better than 0.1 pixels. The World Coordinate System of the WFC3 F336W image was propagated to the other image, to obtain aligned images across filters, and the images in both filters were aligned with north up and east left. The images are in units of $\mathrm{e}^{-} \mathrm{s}^{-1}$, which are converted to physical units using the WFC3 photometric zeropoints, included as keywords in the headers of the data products and posted at: http://www.stsci.edu/hst/wfc3/phot_zp_lbn. The basic details of the LEGUS images for NGC 5253 used in this paper are given in Table 1.

\subsection{Archival Images}

The HST Archive contains a rich collection of images for NGC 5253. For this paper, we retrieved images spanning from the UV to the H-band through the Hubble Legacy Archive ${ }^{41}$ (HLA), both broad and narrow-band, to cover stellar continuum as well as optical and near-IR emission lines. When images in similar bands were available, preference was given to those at the higher angular resolution (e.g., ACS/HRC images were preferred over ACS/WFC images). Because of the extended

\footnotetext{
38 Anderson, J., 2013, http://www.stsci.edu/hst/wfc3/tools/cte_tools

39 http://www.stsci.edu/hst/wfc3/ins_performance/CTE/ ANDERSON_UVIS_POSTFLASH_EFFICACY.pdf

40 see: http://drizzlepac.stsci.edu/

41 http://hla.stsci.edu
} 
Table 1

Characteristics of the HST Images of NGC 5253

\begin{tabular}{|c|c|c|c|c|c|c|c|c|}
\hline $\begin{array}{l}{\text { Instrument } / \text { Camera }^{a}} \\
\text { (1) }\end{array}$ & $\begin{array}{r}\text { Pixel Size }^{\mathrm{a}} \\
(") \\
(2)\end{array}$ & $\begin{array}{r}\text { Ang. Res. } \\
\left(^{a}\right. \\
(3)\end{array}$ & $\begin{array}{l}\text { Field of View } \\
\left(" \times{ }^{\mathrm{a}}\right. \\
(4)\end{array}$ & $\begin{array}{l}\text { Filter }^{\mathrm{b}} \\
(5)\end{array}$ & $\begin{array}{r}\text { Pivot Wavelength }^{\mathrm{b}} \\
(\AA) \\
(6)\end{array}$ & $\begin{array}{r}\text { Exposure Time }^{c} \\
(\mathrm{~s}) \\
(7)\end{array}$ & $\begin{array}{l}\text { Date Obs. }^{\mathrm{d}} \\
\text { (8) }\end{array}$ & $\begin{array}{l}\text { Program }^{\mathrm{d}} \\
\text { (9) }\end{array}$ \\
\hline $\mathrm{ACS} / \mathrm{SBC}$ & 0.030 & 0.097 & $35 \times 31$ & F125LP & 1438.2 & $2660.0(665 . \times 4)$ & 2009 Mar 07 & GO-11579 \\
\hline \multirow[t]{2}{*}{ WFC3/UVIS } & 0.040 & 0.070 & $162 \times 162$ & F275W & 2710.1 & $2448.0(816 . \times 3)$ & $\begin{array}{l}2013 \\
\text { Aug } 28\end{array}$ & GO-13364 \\
\hline & & & & F336W & 3354.8 & $2346.0(782 . \times 3)$ & $\begin{array}{l}2013 \\
\text { Aug } 28\end{array}$ & GO-13364 \\
\hline \multirow[t]{5}{*}{ ACS/HRC } & 0.025 & 0.060 & $29 \times 26$ & F330W & 3362.7 & $1796.0(449 . \times 4)$ & $2006 \mathrm{Feb} 20$ & GO-10609 \\
\hline & & & & F435W & 4311.0 & $600.0(150 . \times 4)$ & $2006 \mathrm{Feb} 20$ & GO-10609 \\
\hline & & & & F550M & 5579.8 & $800.0(200 . \times 4)$ & 2006 Feb 20 & GO-10609 \\
\hline & & & & $\begin{array}{l}\text { F658N } \\
\quad(\mathrm{H} \alpha+[\mathrm{N} \mathrm{II}])\end{array}$ & 6583.7 & $240.0(60 . \times 4)$ & 2006 Feb 20 & GO-10609 \\
\hline & & & & F814W & 8115.4 & $368.0(92 . \times 4)$ & $2006 \mathrm{Feb} 20$ & GO-10609 \\
\hline \multirow[t]{2}{*}{ WFC3/IR } & 0.130 & 0.22 & $123 \times 136$ & F110W & 11534.0 & $597.6(199.2 \times 3)$ & $2011 \mathrm{Jul} 26$ & GO-12206 \\
\hline & & & & $\mathrm{F} 128 \mathrm{~N}(\mathrm{P} \beta)$ & 12832.0 & $1497.6(499.2 \times 3)$ & $2011 \mathrm{Jul} 26$ & GO-12206 \\
\hline \multirow[t]{4}{*}{ NICMOS/NIC2 } & 0.075 & 0.13 & $19 \times 19$ & F110W & 11292.0 & $96.0(24 . \times 4)$ & 1998 Jan 04 & GO-7219 \\
\hline & & & & F160W & 16071.0 & $96.0(24 . \times 4)$ & 1998 Jan 04 & GO-7219 \\
\hline & & & & $\mathrm{F} 187 \mathrm{~N}(\mathrm{P} \alpha)$ & 18747.8 & $256.0(64 . \times 4)$ & 1998 Jan 04 & GO-7219 \\
\hline & & & & F190N & 18986.0 & $256.0(64 . \times 4)$ & 1998 Jan 04 & GO-7219 \\
\hline
\end{tabular}

Notes.

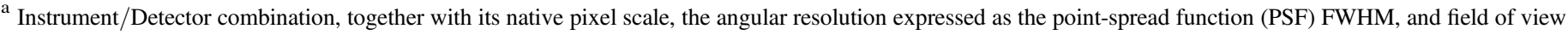

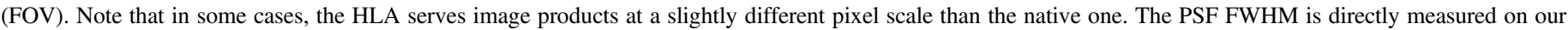
images.

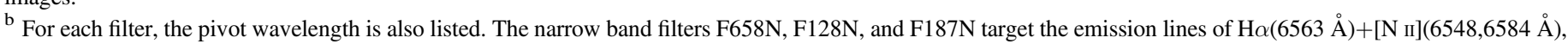
$\mathrm{P} \beta(12818 \AA)$, and $\mathrm{P} \alpha(18756 \AA)$, respectively. The F190N narrow-band image is used for the stellar continuum subtraction of the F187N image.

c The total exposure time, shown in units of seconds, is usually the result of 3 or 4 combined (dithered) exposures, with individual times as indicated.

${ }^{\mathrm{d}}$ Date in which the observation took place, and name of the HST observing program that obtained the data.

wavelength coverage, the images used here have been obtained with different $H S T$ instruments, including the ACS/SBC, ACS/HRC, WFC3/IR, and NICMOS. Level 2 products were retrieved for each instrument/filter combination, implying that the individual post-pipeline exposures have been aligned, cosmic-ray cleaned, and combined using either MultiDrizzle or ASTRODRIZZLE. The retrieved images have also been geometrically corrected and aligned with north up and east left. All images are provided by the HLA in units of $\mathrm{e}^{-} \mathrm{s}^{-1}$. We convert all images, except those from NICMOS, to physical units using the photometric zero points appropriate for each instrument/ filter combination. The photometric zero points of the NICMOS images are referred to the default calibration of the instrument in $\mathrm{DN} \mathrm{s}^{-1}$, thus we divide the HLA images first by the NICMOS Camera 2 gain $\left(5.4 \mathrm{e}^{-} / \mathrm{DN}\right)$ in order to apply the published zero points. The details for each image product are listed in Table 1.

Although the archival data display a range of depths (as indicated by the large range of exposure times in Table 1), all sources we study are detected with signal-to-noise ratio $(\mathrm{S} / \mathrm{N})$ $>100$ in the broad and medium band filters. The uncertainty in the photometry is driven by crowding and uncertainties in the aperture corrections, rather than $\mathrm{S} / \mathrm{N}$ limitations.

\subsection{Additional Processing}

Improved alignment of all the images, both new and archival, is accomplished using the IRAF ${ }^{42}$ tasks geomap and

\footnotetext{
${ }^{42}$ IRAF is distributed by the National Optical Astronomy Observatory, which is operated by the Association of Universities for Research in Astronomy (AURA) under a cooperative agreement with the National Science Foundation.
}

geotran and a sample of stellar sources in the ACS/HRC images as reference. The HRC images are preferred over others, because they have the smallest native pixel, which, in this case, drives the angular resolution of the final images. Thus, we elect to preserve as much as possible the highest angular resolution, even if it results in oversampling some of the lower resolution images. For the same reason, the aligned images are all re-sampled to the pixel scale of the ACS/HRC, 0 ."025 pixel $^{-1}$ (Table 1).

After alignment, all images dominated by stellar continuum, i.e., all filters except F658N, F129N, and F187N, are converted to physical units of erg s${ }^{-1} \mathrm{~cm}^{-2} \AA^{-1}$ using the most up-to-date values of the PHOTFLAM keyword as posted on the relevant webpage for each instrument (see example for WFC3 in previous section).

The nebular continuum and line emission from the central radio nebula significantly contaminate the fluxes in the broad band filters, for the clusters both within the nebula and in the surrounding region. For instance, the presence of line emission in the $\mathrm{F} 814 \mathrm{~W}$ filter increases the flux measured for our individual sources between a few percent and a factor $>3$, depending on the location of the source. This effect has been noted as a problem for measurements of young sources by others (Johnson et al. 1999; Reines et al. 2010). Contamination by emission lines of broad band filters, in turn, affects the derivation of the line flux intensities themselves, since the broad band images are used for the subtraction of the stellar continuum from narrow band images. Nebular continuum will not have the same effect, since it is present in both broad and narrow band filters. 
We derive emission-line-free images for the most affected among our filters: F435W, F814W, and F110W. The F110W filter receives most of the emission line contribution from $\mathrm{P} \beta$, and we use iterative subtraction between the F110W and F128N filters to remove the line contamination. For the lines affecting the F435W and F814W filters we do not have direct imaging in the corresponding narrow-band filters. We thus use the 3200-10,000 $\AA$ spectrum of Storchi-Bergmann et al. (1995) of the central $10^{\prime \prime} \times 20^{\prime \prime}$ region of NGC 5253 convolved with the $\mathrm{F} 435 \mathrm{~W}$ and $\mathrm{F} 814 \mathrm{~W}$ transmission curves to estimate the emission line contamination in these filters. The spectrum by Storchi-Bergmann et al. (1995) covers a sizable fraction of the region of interest here, along the $\mathrm{E}-\mathrm{W}$ direction, and is thus representative of the excitation conditions in the center of NGC 5253. The H $\alpha$ image derived from the F658N filter (see below) is then rescaled to the intensity of the emission lines and subtracted from both the F435W and F814W images. This process converges within two iterations. The remaining broad and medium band images are not significantly contaminated by emission lines, as estimated from the same spectrum.

Emission line images are derived directly from the narrowband filters, after subtracting the underlying stellar and nebular continuum. All narrow-band filters are converted to monochromatic fluxes (erg s${ }^{-1} \mathrm{~cm}^{-2} \AA^{-1}$ ), before performing continuum subtraction. The continuum images are derived as follows. For the F128N image, which contains the $\mathrm{P} \beta$ line, the rescaled, nebular-line-subtracted F110W image is used. Although straightforward, this method can include hard-toquantify uncertainties, if there are significant color changes in the stellar population across the field of view. For the F187N image, which contains the $\mathrm{P} \alpha$ line, we employ the rescaled F190N narrow-band image (Table 1), which is free of emission lines and of any complications induced by potential color changes across the field. For both the F110W and F190N images, the rescaling factors are determined from emission-free point sources. For the F658N filter, which contains $\mathrm{H} \alpha+[\mathrm{N}$ II $]$, we create a continuum image by interpolating the fluxcalibrated F550M and line-emission-subtracted F814W images. The resulting image is then subtracted from the flux-calibrated F658N image, without rescaling.

The line emission images are then converted to units of $\operatorname{erg~s}^{-1} \mathrm{~cm}^{-2}$ by: (a) multiplying each image by the filter bandpass $^{43}$ (72, $159 \AA$, and $188 \AA$, for F658N, F128N, and F187N, respectively); and (b) correcting for the filter transmission curve values at the location of the redshifted lines. We remove the $\left[\mathrm{N}\right.$ II] emission from the $\mathrm{F} 658 \mathrm{~N}$ image using $\left[\mathrm{N}_{\mathrm{II}}\right]$ $(6584 \AA) / \mathrm{H} \alpha=0.084$ from Moustakas \& Kennicutt (2006), which is close to the value obtained from the spectrum of Storchi-Bergmann et al. (1995), and the atomic ratio [N $\mathrm{NI}]$ $(6548 \AA) /[\mathrm{N}$ II $](6584 \AA)=0.3$.

\section{CLUSTER SELECTION AND PHOTOMETRY}

Two cluster candidates are selected within the radio nebula (Figures 1(a) and 2): one corresponding to the observed peak in $\mathrm{H} \alpha$ and the other corresponding to the observed peak in $\mathrm{P} \alpha$. Measurements at $7 \mathrm{~mm}$ indicate a size of $\sim 1$ !' $2(\sim 18 \mathrm{pc})$ for the radio nebula (Turner \& Beck 2004), as shown by the orange circle in the left-hand-size panel of Figure 2. The two peaks,

\footnotetext{
43 The filter bandpass is defined as the filter rectangular width, i.e., the equivalent width divided by the maximum throughput within the filter bandpass. See, e.g., http://www.stsci.edu/hst/wfc3/documents/handbooks/ currentIHB/c07_ir06.html.
}

$\mathrm{H} \alpha$ and $\mathrm{P} \alpha$, are separated by about 0 ". 46 mainly along the $\mathrm{E}-\mathrm{W}$ direction (Figure 2), corresponding to a spatial separation of $\sim 7 \mathrm{pc}$, with the $\mathrm{P} \alpha$ peak emission located to the west of the $\mathrm{H} \alpha$ one. For each peak, the other line is also present, but not as prominently. The $\mathrm{H} \alpha$ peak, called " 5 " in Figure 1(a), has both $\mathrm{P} \beta$ and $\mathrm{P} \alpha$ emission spatially coincident with each other, and also with the continuum emission, within the accuracy that can be established from the image-to-image resolution differences (column 3 of Table 1).

The cluster candidate corresponding to the $\mathrm{P} \alpha$ peak, called "11" in Figure 1(a), is slightly offset, by about 0 ". $1(\sim 1.5 \mathrm{pc})$, to the east of the centroid of the $\mathrm{H} \alpha$ emission closest to it, while the $\mathrm{P} \beta$ centroid falls in-between the peak locations of the other two lines. ${ }^{44}$ This gradual transition as a function of increasing wavelength suggests that the offsets between the peaks of the hydrogen emission lines are likely due to variations in the dust optical depth, rather than the presence of separate sources of emission. Although the latter scenario cannot be completely ruled out, we will assume in this work that the slightly spatially shifted lines all originate from the same source. A visual inspection of the continuum images shows that the spatial shift occurs between the $\mathrm{J}(\mathrm{F} 110 \mathrm{~W})$ and $\mathrm{H}(\mathrm{F} 160 \mathrm{~W})$ images, and no shift is obviously present at shorter wavelengths; the centroid of the source in the NICMOS F110W image coincides with the centroids in the shorter wavelength images, while the centroid in the NICMOS F160W image coincides with the centroid of the $\mathrm{P} \alpha$ peak.

Both clusters 5 and 11 are close to the peaks of free-free emission at $\mathrm{cm}$ wavelengths studied by Turner et al. (2000) (Figure 2). Cluster 11 is within 0!'18, toward the S-W direction, of the peak at both 1.3 and $2 \mathrm{~cm}$, while cluster 5 is $\sim 0$ ". 18 to the south of the secondary peak at $1.3 \mathrm{~cm}$. The coincidence between the sources would be increased if the relative astrometry between the $H S T$ and the cm-wavelength observations were off by about 0. ". 2 along the $\mathrm{N}-\mathrm{S}$ direction. This is consistent with the 0 ." $1-0$ ". 3 uncertainty of the absolute astrometry for HST images (e.g., Koekemoer et al. 2006). We thus believe the optical and radio peaks to be actually coincident, in agreement with the assumption of AlonsoHerrero et al. (2004); the observed offsets are likely due to small errors in the absolute reference frames of the two sets of data.

An additional nine star clusters, all visually identified as local peaks of emission in the $V(\mathrm{~F} 550 \mathrm{M})$ band and all brighter than $m_{V}=18.7 \mathrm{mag}\left(M_{V}<-8.8 \mathrm{mag}\right)$, are selected in order to perform tests on the SED fitting approach we adopt for this study. We ensure that the selected sources are clusters by requiring that each source's FWHM is at least 50\% broader than the stellar PSF. Our compilation brings the total number of star clusters investigated here to 11 , whose locations are identified in Figure 1(a) and best-fit ages in Figure 1(b) (see next section).

Photometry is performed for all 11 clusters in multiple ways. Our default photometry uses an aperture of 5 pixels radius $(0$ !' $125 \sim 1.9 \mathrm{pc})$ with the background measured in an annulus with inner radius of 20 and 3 pixels wide. We perform visual inspection of the sky annuli for each cluster to ensure that they are not affected by contamination from surrounding bright stars/clusters. We also run tests using sky annuli with inner

\footnotetext{
44 Centroids of local emission peaks can be determined with an accuracy of about $1 / 5$ th-1/10th pixel, which, for the low-resolution WFC3/IR images, corresponds to a location accuracy of better than $\sim 0$. $^{\prime} 02$.
} 

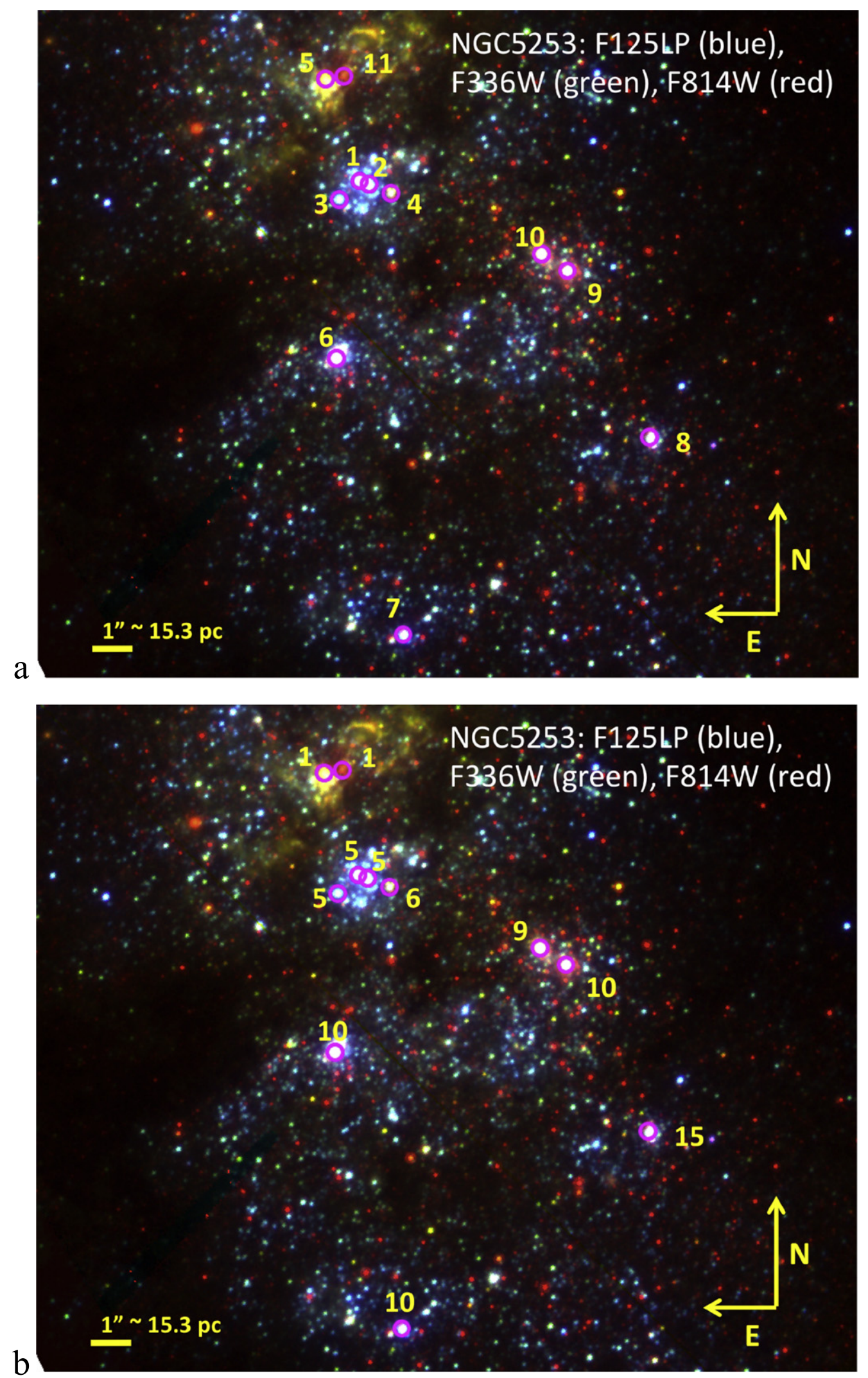

Figure 1. (a) A three-color composite of the central $20^{\prime \prime} \times 16^{\prime \prime}(\sim 300 \times 250 \mathrm{pc})$ of NGC 5253, combining the ACS/SBC/F125LP (blue), WFC3/UVIS/F336W (green), and ACS/HRC/F814W (red) bands. The 11 star clusters in this study are identified with magenta circles and numbered. The circle radii are 7.5 pixels ( 0 "' 19 $\sim 2.9 \mathrm{pc}$ ), i.e., $50 \%$ larger than the baseline photometric apertures used in this work. Clusters 5 and 11 are located within the central radio emission region (see Figure 2 and Turner et al. 2000). A ruler of $1^{\prime \prime}$ size $(\sim 15.3 \mathrm{pc})$ is provided at the bottom-left of the figure. (b) The same as Figure 1, where the clusters' labels have been replaced by their best-fit ages. 

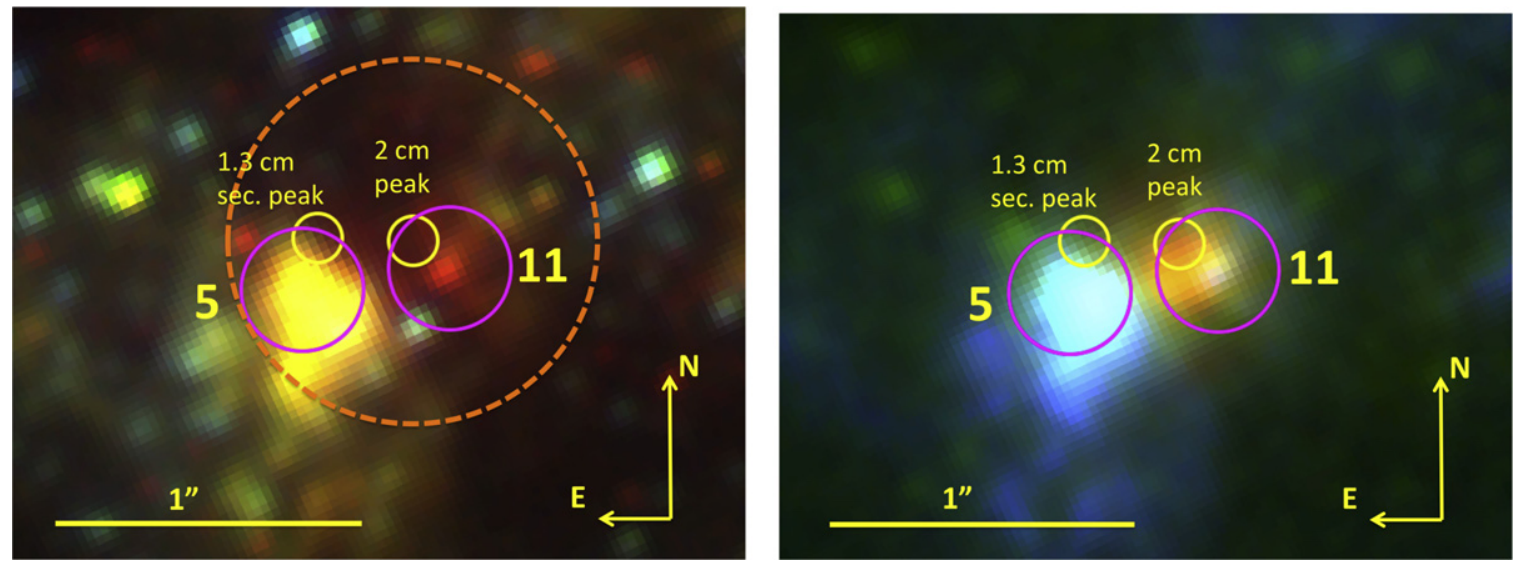

Figure 2. Detail of the region surrounding clusters 5 and 11, using (left) the same color composite as in Figure 1 and (right) a three color-composite using the H $\alpha$ emission line (blue), the NICMOS/NIC2/F110W (green), and the P $\alpha$ emission line (red). The region has size 2 !" $4 \times 1$ !" $8(\sim 37 \times 27$ pc). The two clusters are located at the center of the magenta circles, in correspondence of the $\mathrm{H} \alpha$ (cluster 5) and the $\mathrm{P} \alpha$ (cluster 11) emission peaks, respectively. The yellow circles identify the nominal positions, relative to the astrometry of the LEGUS WFC 3 images, of the peak at 1.3 and $2 \mathrm{~cm}$ (right-hand side circle) and the secondary peak at $1.3 \mathrm{~cm}$ (lefthand side circle) identified by Turner et al. (2000). The absolute astrometric uncertainty of the HST images, $0.11-0.13$, is comparable to the radius of the magenta circles $(0$. . 2). The diameters of the yellow circles, 0. . 15, are comparable to the size of the mm and cm beams (Turner et al. 2000; Turner \& Beck 2004). The orange circle in the left-hand side panel marks the extent of the $7 \mathrm{~mm}$ emitting region, 1 ." 2 (Turner \& Beck 2004), which we term the "radio nebula." The color-composite in the right-hand side panel highlights: (1) the differential intensity of the $\mathrm{H} \alpha$ and $\mathrm{P} \alpha$ emission in correspondence of the two clusters, with $\mathrm{H} \alpha$ being stronger in cluster 5 and $\mathrm{P} \alpha$ being stronger in cluster 11; and (2) the color gradient shown by the emission lines in cluster 11. The emission map in $\mathrm{P} \beta$ is not shown, because of the significantly lower angular resolution of the WFC3/IR images relative to the ones shown here.

radius in the range 15-20 pixels and width in the range 3-6 pixels, in order to quantify the effects of background contamination. The resulting photometry varies by less than $8 \%$, a much smaller uncertainty than those introduced by other effects (e.g., aperture corrections) as discussed below. For the broad-band filters, we perform photometry on both the nebularline subtracted and unsubtracted images, which we will compare to appropriate synthetic photometry from stellar population synthesis models (with and without nebular line emission, see next section). For the emission lines, we perform photometry on the stellar-continuum subtracted images.

As some of the clusters show a complex structure (typically elongated), we also perform larger-aperture photometry, with 10-15 pixels radii (and up to 20 pixels for the emission lines), for the more spatially isolated clusters. We use this larger aperture photometry as a check for our aperture corrections, especially for the WFC3/IR images, which have pixel size comparable to the radius of the default photometric aperture. We choose not to adopt the larger radius apertures as default for photometry, because a few of the 11 star clusters, including both clusters in the radio nebula, are located in crowded regions.

The aperture corrections are determined from isolated star clusters found around the region where our 11 target clusters are located. We derive separate corrections for each instrument/filter combination. For the medium/broad-band filters (stellar continuum), the aperture corrections needed to bring the 5-pixel radius photometry to the infinite-aperture equivalent range from a factor 1.7 (WFC3/UVIS/F275W) to a factor 2.25 (NICMOS/NIC2/F160W), with a larger value, 2.81, for the WFC3/IR/F110W instrument/filter combination. For the emission lines, the aperture corrections for a 5-pixel radius are significantly larger, between a factor 3.7 and 7.8, which accounts for the more extended nature of the nebular emission. As expected, the aperture corrections decrease significantly, with values ranging from $5 \%$ to $20 \%$ for the stellar continuum filters, and from $40 \%$ to $70 \%$ for the emission lines, when a 15-pixel radius aperture is used for photometry. Comparisons between our default aperture and larger-aperture photometry indicates uncertainties of $\sim 15 \%$ for all UV-optical medium/ broad-band filters, $20 \%$ for the NICMOS stellar continuum filters, and $35 \%$ for the WFC $3 / \mathrm{IR} / \mathrm{F} 110 \mathrm{~W}$ filter; for the emission lines, we derive: $\sim 30 \%-55 \%-35 \%$ uncertainty for $\mathrm{H} \alpha, \mathrm{P} \beta$, and $\mathrm{P} \alpha$, respectively. The larger uncertainty for the WFC3/IR photometry simply reflects the larger pixel scale of these images. The aperture corrections are the largest source of uncertainty for the stellar continuum filters; the emission lines suffer from an additional (smaller) uncertainty due to the underlying stellar continuum subtraction. Together with small registration offsets, this is especially a limitation for the $\mathrm{P} \beta$ photometry, despite having one of the deepest among our exposures. The shallow depth of the exposure is an additional limitation for the $\mathrm{P} \alpha$ image (Table 1). The combination of all uncertainties, excluding the aperture correction ones, gives $1 \sigma$ depths of: $L(\mathrm{H} \alpha)=2.3 \times 10^{35} \mathrm{erg} \mathrm{s}^{-1}, L(\mathrm{P} \beta)=$ $3.5 \times 10^{35} \mathrm{erg} \mathrm{s}^{-1}$, and $L(\mathrm{P} \alpha)=2.8 \times 10^{35} \mathrm{erg} \mathrm{s}^{-1}$.

All photometry is corrected for foreground Milky Way extinction, using the extinction curve values listed in Table 2 and the color excess $E(B-V)=0.049$ from Schlafly \& Finkbeiner (2011, as retrieved from the NASA/IPAC Extragalactic Database). The values of Table 2 can be directly applied to photometric measurements only for small values of the color excess, typically $E(B-V) \lesssim 0.1$, since color variations across the filter bandpass are typically small; for larger values of the color excess, the extinction correction should be applied to the source's SED before convolution with the telescope/ instrument/filter response curve.

Table 3 lists the 11 star clusters, their coordinates, and the cross-IDs with other studies (Calzetti et al. 1997; Harris et al. 2004; de Grijs et al. 2013), where available, ${ }^{45}$ together with photometry, $\mathrm{H} \alpha$ equivalent widths $(\mathrm{EW})$, and the color excess as inferred from the hydrogen emission line ratios. The listed photometry is for the measurements performed in the

\footnotetext{
45 Our coordinates are slightly offset relative to those of de Grijs et al. (2013) by $\Delta \alpha=-0.057 \mathrm{~s}$ and $\Delta \delta=-0$." 15 .
} 
Table 2

Foreground Extinction Correction Values

\begin{tabular}{lrc}
\hline \hline Filter & Band & $k(\lambda)$ \\
\hline F125LP & FUV & 8.54 \\
F275W & NUV & 6.29 \\
F336W & $\mathrm{U}$ & 5.07 \\
F330W & $\mathrm{U}$ & 5.06 \\
F435W & $\mathrm{B}$ & 4.21 \\
F550M & $\mathrm{V}$ & 3.05 \\
F658N & $\mathrm{H} \alpha$ & 2.54 \\
F814W & $\mathrm{I}$ & 1.80 \\
F110W(NIC2) & $\mathrm{J}$ & 1.03 \\
F110W(WFC3) & $\mathrm{J}$ & 1.00 \\
F128N & $\mathrm{P} \beta$ & 0.84 \\
F160W & $\mathrm{H}$ & 0.58 \\
F187N & $\mathrm{P} \alpha$ & 0.46 \\
\hline
\end{tabular}

Note.

a The extinction curve for the Milky Way, $k(\lambda)$, expressed as: $F_{\text {obs }}(\lambda)=F_{\text {int }}(\lambda)$ $10^{-0.4 E(B-V) k(\lambda)}$. The values of the extinction curve are from the parametrization of Fitzpatrick (1999), with total-to-selective extinction value $R_{V}=3.1$.

5-pixel apertures, corrected for foreground Milky Way extinction and for aperture effects; in the case of continuum images, the photometry is from the original images, which include contribution from emission lines. Overall, the photometry of cluster 11 has larger uncertainties than that of the other clusters in our sample, due to its low flux densities, which are from a few times to over an order of magnitude fainter, depending on wavelength.

The EWs of $\mathrm{H} \alpha$, calculated from the ratio of the emission line flux to the stellar continuum flux density (interpolated from emission-line-subtracted images, see previous section), are given as a range: the smaller value corresponds to the ratio of line-to-continuum for measurements within the 5-pixel radius aperture; the larger value corresponds to the ratio obtained after both line and continuum have been corrected for aperture effects. The color excess values are derived from the line ratios $\mathrm{H} \alpha / \mathrm{P} \beta$ and $\mathrm{H} \alpha / \mathrm{P} \alpha$ using the selective extinction values that can be derived from Table 2, i.e., $k(\mathrm{H} \alpha)-k(\mathrm{P} \beta)=1.70$ and $k(\mathrm{H} \alpha)-k(\mathrm{P} \alpha)=2.08$, and the simple assumption of a foreground dust screen. For the intrinsic line ratios we adopt $\mathrm{H} \alpha / \mathrm{P} \beta=17.57$ and $\mathrm{H} \alpha / \mathrm{P} \alpha=8.64$, which are appropriate for $\mathrm{H}$ II regions with electron temperature $T_{\mathrm{e}} \sim 11,500 \mathrm{~K}$, measured for NGC 5253 (Lopez-Sanchez et al. 2007). We do not report the color excess derived from the ratio $\mathrm{P} \beta / \mathrm{P} \alpha$, since the selective extinction between the wavelengths of these two lines is small, and thus the resulting colors excess is subject to large uncertainties.

Two sets of values correspond to measurements performed at similar or close wavelengths, but with different instruments (Table 1): the WFC3/UVIS/F336W and the WFC3/IR/ F110W measurements can be compared with the ACS/HRC/ F330W and NICMOS/NIC2/F110W measurements, respectively. A close inspection of the photometry listed in Table 3 shows that the photometry in the two blue filters, WFC3/ UVIS/F336W and ACS/HRC/F330W, is usually comparable to better than $15 \%(\sim 0.07$ in log scale $)$, with the exception of cluster 11 , where the difference is about $25 \%(\sim 0.1$ in $\log$ scale). We attribute the discrepancy to the difficulty of determining the background level around this highly obscured star cluster; however, even in this case the difference in photometry is still within the combined $1 \sigma$ error of the two measurements. Conversely, the photometric values in WFC3/ IR/F110W and NICMOS/NIC2/F110W tend to be more discrepant with each other, with differences that range from $10 \%$ to $40 \%$ ( $0.04-0.15$ in log scale). There is no obvious trend for one measurement to be systematically higher or lower than the other, although the NICMOS/NIC2/F110W measurement is more frequently the lower value. As the NICMOS /NIC2/ F110W filter is at slightly shorter wavelength than the WFC3/ IR/F110W, its photometry values should be higher, thus the observed discrepancy is likely a combination of measurement uncertainties and, possibly, some systematic calibration offset. Similarly to the other pair of filters, the discrepancies are within the combined $1 \sigma$ error of the two measurements.

Color-color plots of the 11 clusters in selected bands are shown in Figure 3, together with the tracks of model stellar populations (Section 4). These plots are only shown to guide intuition, and will not be used to derive the physical properties of the star clusters.

\section{SYNTHETIC PHOTOMETRY AND FITTING APPROACH}

Spectral energy distributions (SEDs) from the UV to the near-IR are generated using the Starburst99 (Leitherer et al. 1999, version as available in early 2014) spectral synthesis models, using instantaneous star formation, with a Kroupa (2001) IMF in the range $0.1-120 M_{\odot}$ and metallicity $Z=0.004$ ( $\sim 30 \%$ solar), which is the closest value to the measured oxygen abundance of NGC 5253 and for which models are available. We produce models using both the Padova with AGB treatment ${ }^{46}$ and the Geneva tracks (Meynet et al. 1994; Girardi et al. 2000; Vazquez \& Leitherer 2005). Since the clusters under consideration tend to be massive, $M \gtrsim 10^{4} M_{\odot}$, we expect minimal impact from stochastic sampling of the IMF (Cerviño \& Luridiana 2004), and use the default deterministic models. The Starburst99 models include nebular continuum, but not nebular emission lines. These are added by Yggdrasil (Zackrisson et al. 2011), which uses Starburst99 stellar populations as an input for CLOUDY (Ferland et al. 2013). For Yggdrasil, we adopt a 50\% covering factor for the ionized gas, meaning that only $50 \%$ of the nebular emission is spatially coincident with the star cluster. This attempts to reproduce the observed trend for nebular emission to be more extended than the stellar continuum (see previous section). Models with and without emission lines are generated for the age range $1 \mathrm{Myr}-$ $1 \mathrm{Gyr}$ in steps of $1 \mathrm{Myr}$ in the $1-15 \mathrm{Myr}$ range, $10 \mathrm{Myr}$ in the 20-100 Myr range, and $100 \mathrm{Myr}$ in the 200-1000 Myr range. Instantaneous models are assumed here to reasonably represent the population of individual star clusters.

The SEDs produced by both Starburst99 and Yggdrasil are attenuated with: a starburst attenuation curve (Calzetti et al. 2000), and a Milky Way, an LMC and an SMC extinction curve (as parametrized by Fitzpatrick 1999). For the extinction curves, we adopt a foreground dust geometry (Calzetti 2001) of the form:

$$
F(\lambda)_{\text {out }}=F(\lambda)_{\text {model }} 10^{[-0.4 E(B-V) k(\lambda)]},
$$

and both cases of equal and differential attenuation for the nebular gas and stellar continuum; for the differential attenuation, we assume that the stellar continuum is subject

\footnotetext{
$\overline{46}$ Our clusters are young enough, $\lesssim 15 \mathrm{Myr}$, that use of the Padova tracks without AGB treatment yields identical results.
} 
Table 3

Cluster Location and Photometry

\begin{tabular}{|c|c|c|c|c|c|c|c|c|c|c|c|}
\hline $\begin{array}{l}\text { Field } \\
\text { (1) }\end{array}$ & $\begin{array}{r}\# 1 \\
(2)\end{array}$ & $\begin{array}{r}\# 2 \\
\text { (3) }\end{array}$ & $\begin{array}{r}\# 3 \\
\text { (4) }\end{array}$ & $\begin{array}{r}\# 4 \\
(5)\end{array}$ & $\begin{array}{r}\# 5 \\
(6)\end{array}$ & $\begin{array}{r}\# 6 \\
(7)\end{array}$ & $\begin{array}{r}\# 7 \\
(8)\end{array}$ & $\begin{array}{r}\# 8 \\
(9)\end{array}$ & $\begin{array}{l}\# 9 \\
(10)\end{array}$ & $\begin{array}{r}\# 10 \\
(11)\end{array}$ & $\begin{array}{r}\# 11 \\
(12)\end{array}$ \\
\hline $\begin{array}{l}\text { R.A. }(J 2000)^{a} \\
\end{array}$ & $13: 39: 55.919$ & $13: 39: 55.901$ & $13: 39: 55.960$ & $13: 39: 55.858$ & $13: 39: 55.986$ & $13: 39: 55.965$ & $13: 39: 55.833$ & 13:39:55.351 & $13: 39: 55.512$ & $13: 39: 55.563$ & $13: 39: 55.951$ \\
\hline $\operatorname{Decl}(\mathrm{J} 2000)^{\mathrm{a}}$ & $-31: 38: 27.09$ & $-31: 38: 27.18$ & $-31: 38: 27.57$ & $-31: 38: 27.41$ & $-31: 38: 24.54$ & $-31: 38: 31.51$ & $-31: 38: 38.49$ & $-31: 38: 33.54$ & $-31: 38: 29.35$ & $-31: 38: 28.92$ & $-31: 38: 24.45$ \\
\hline Cross-IDs ${ }^{\mathrm{b}}$ & $4,4,95$ & $4,4,90 ?$ & $\ldots, 8,106$ & $\ldots, \ldots, \ldots$ & $5,1,87$ & $1,2,129$ & $\ldots, 27,156$ & $6,9,36$ & $3,3,38$ & $2,5,45$ & $\ldots, \ldots, .$. \\
\hline$L(\mathrm{~F} 125 \mathrm{LP})^{\mathrm{c}}$ & $36.56(0.07)$ & $36.68(0.07)$ & $36.38(0.07)$ & $35.91(0.07)$ & $36.00(0.07)$ & $36.66(0.07)$ & $36.52(0.07)$ & $36.28(0.07)$ & $36.30(0.07)$ & $36.08(0.07)$ & $34.66(0.16)$ \\
\hline$L(\mathrm{~F} 275 \mathrm{~W})^{\mathrm{c}}$ & $36.07(0.07)$ & $36.14(0.07)$ & $35.88(0.07)$ & $35.71(0.07)$ & $35.79(0.07)$ & $36.20(0.07)$ & $35.92(0.07)$ & $35.81(0.07)$ & $35.92(0.07)$ & $35.73(0.07)$ & $34.23(0.12)$ \\
\hline$L(\mathrm{~F} 336 \mathrm{~W})^{\mathrm{c}}$ & $35.87(0.07)$ & $35.93(0.07)$ & $35.67(0.07)$ & $35.58(0.07)$ & $35.84(0.07)$ & $35.98(0.07)$ & $35.70(0.07)$ & $35.65(0.07)$ & $35.77(0.07)$ & $35.60(0.07)$ & $34.36(0.09)$ \\
\hline$L(\mathrm{~F} 330 \mathrm{~W})^{\mathrm{c}}$ & $35.87(0.07)$ & $35.90(0.07)$ & $35.66(0.07)$ & $35.63(0.07)$ & $35.82(0.07)$ & $35.97(0.07)$ & $35.67(0.07)$ & $35.62(0.07)$ & $35.75(0.07)$ & $35.59(0.07)$ & $34.45(0.09)$ \\
\hline$L(\mathrm{~F} 435 \mathrm{~W})^{\mathrm{c}}$ & $35.62(0.07)$ & $35.64(0.07)$ & $35.41(0.07)$ & $35.42(0.07)$ & $35.63(0.07)$ & $35.77(0.07)$ & $35.44(0.07)$ & $35.51(0.07)$ & $35.67(0.07)$ & $35.66(0.07)$ & $34.25(0.15$ \\
\hline$L(\mathrm{~F} 550 \mathrm{M})^{\mathrm{c}}$ & $35.36(0.07)$ & $35.35(0.07)$ & $35.15(0.07)$ & $35.25(0.07)$ & $35.30(0.07)$ & $35.59(0.07)$ & $35.23(0.07)$ & $35.31(0.07)$ & $35.62(0.07)$ & $35.55(0.07)$ & $34.06(0.13)$ \\
\hline$L(\mathrm{~F} 814 \mathrm{~W})^{\mathrm{c}}$ & $34.88(0.07)$ & $34.86(0.07)$ & $34.61(0.07)$ & $34.93(0.07)$ & $35.38(0.07)$ & $35.39(0.07)$ & $35.00(0.07)$ & $34.99(0.07)$ & $35.47(0.07)$ & $35.24(0.07)$ & $34.50(0.10)$ \\
\hline$L(\mathrm{~N} / \mathrm{F} 110 \mathrm{~W})^{\mathrm{c}}$ & $34.35(0.10)$ & $34.34(0.10)$ & $33.84(0.10)$ & $34.45(0.10)$ & $35.15(0.10)$ & $35.18(0.10)$ & $34.66(0.10)$ & $34.66(0.10)$ & $35.21(0.10)$ & $34.90(0.10)$ & $34.81(0.11)$ \\
\hline$L(\mathrm{~W} / \mathrm{F} 110 \mathrm{~W})^{\mathrm{c}}$ & $34.44(0.14)$ & $34.42(0.14)$ & $33.69(0.14)$ & $34.36(0.13)$ & $35.23(0.14)$ & $35.27(0.13)$ & $34.73(0.14)$ & $34.78(0.14)$ & $35.16(0.13)$ & $34.96(0.14)$ & $34.93(0.19)$ \\
\hline$L(\mathrm{~F} 160 \mathrm{~W})^{\mathrm{c}}$ & $33.77(0.10)$ & $33.78(0.10)$ & $33.45(0.10)$ & $34.13(0.10)$ & $34.79(0.10)$ & $35.02(0.10)$ & $34.50(0.10)$ & $34.46(0.10)$ & $35.03(0.10)$ & $34.66(0.10)$ & $34.78(0.11)$ \\
\hline$L(\mathrm{H} \alpha)^{\mathrm{d}}$ & $38.26(0.11)$ & $38.06(0.11)$ & $37.04(0.11)$ & $37.63(0.11)$ & $38.96(0.11)$ & $<35.36$ & $36.77(0.11)$ & $\lesssim 35.36$ & $37.00(0.11)$ & $36.17(0.13)$ & $38.17(0.11)$ \\
\hline$L(\mathrm{P} \beta)^{\mathrm{d}}$ & $37.17(0.19)$ & $37.01(0.19)$ & $36.08(0.21)$ & $36.62(0.19)$ & $38.05(0.19)$ & $<35.54$ & $36.01(0.22)$ & $<35.54$ & $37.22(0.19)$ & $<35.54$ & $37.93(0.19)$ \\
\hline$L(\mathrm{P} \alpha)^{\mathrm{d}}$ & $37.25(0.13)$ & $37.08(0.13)$ & $35.90(0.17)$ & $36.78(0.13)$ & $38.42(0.13)$ & $35.92(0.17)$ & $35.86(0.18)$ & $36.01(0.16)$ & $36.97(0.13)$ & $35.94(0.17)$ & $38.52(0.13)$ \\
\hline $\mathrm{EW}(\mathrm{H} \alpha)^{\mathrm{e}}$ & $450-1140$ & $290-730$ & $40-110$ & $200-300$ & $>3200$ & 0 & $30-40$ & $\approx 1$ & $10-30$ & $\sim 5$ & $>3200$ \\
\hline$E(B-V)_{\mathrm{H} \alpha / P \beta}{ }^{\mathrm{f}}$ & $0.23(0.32)$ & $0.29(0.32)$ & $0.43(0.35)$ & $0.35(0.32)$ & $0.49(0.32)$ & $\ldots$ & $0.71(0.36)$ & $\ldots$ & $2.16(0.32)$ & $\ldots$ & $1.48(0.32)$ \\
\hline$E(B-V)_{\mathrm{H} \alpha / P \alpha}{ }^{\mathrm{f}}$ & $0.00(0.20)$ & $0.00(0.20)$ & $0.00(0.24)$ & $0.10(0.20)$ & $0.47(0.20)$ & $\ldots$ & $0.03(0.25)$ & $1.92(1.22)$ & $1.10(0.20)$ & $0.86(0.26)$ & $1.54(0.20)$ \\
\hline
\end{tabular}

Notes.

${ }^{a}$ Astrometry obtained from the LEGUS WFC3/UVIS/F336W image.

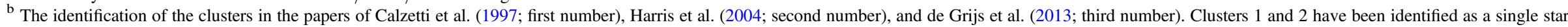

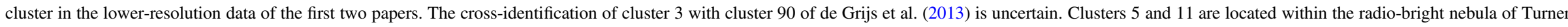
et al. (2000) and Turner \& Beck (2004), see Figure 2.

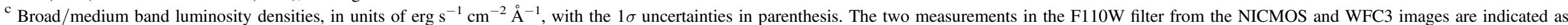

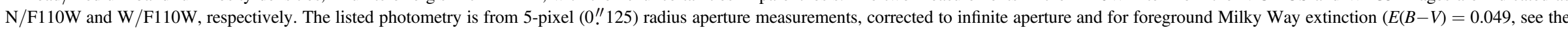
text). The luminosity densities are calculated adopting a distance of $3.15 \mathrm{Mpc}$ for NGC 5253 .

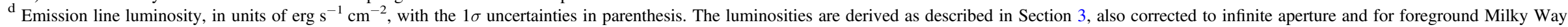
extinction $(E(B-V)=0.049$, see the text), and calculated adopting a distance of $3.15 \mathrm{Mpc}$.

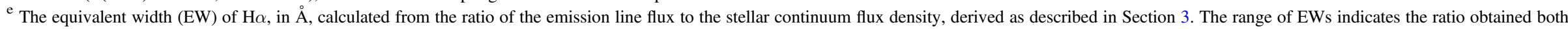
before (smaller value) and after (larger value) aperture correction.

${ }^{\mathrm{f}}$ The color excess derived from the indicated emission lines, under the simple assumption of a foreground dust screen, using the selective extinction values from Table 2. See the text for more details. 

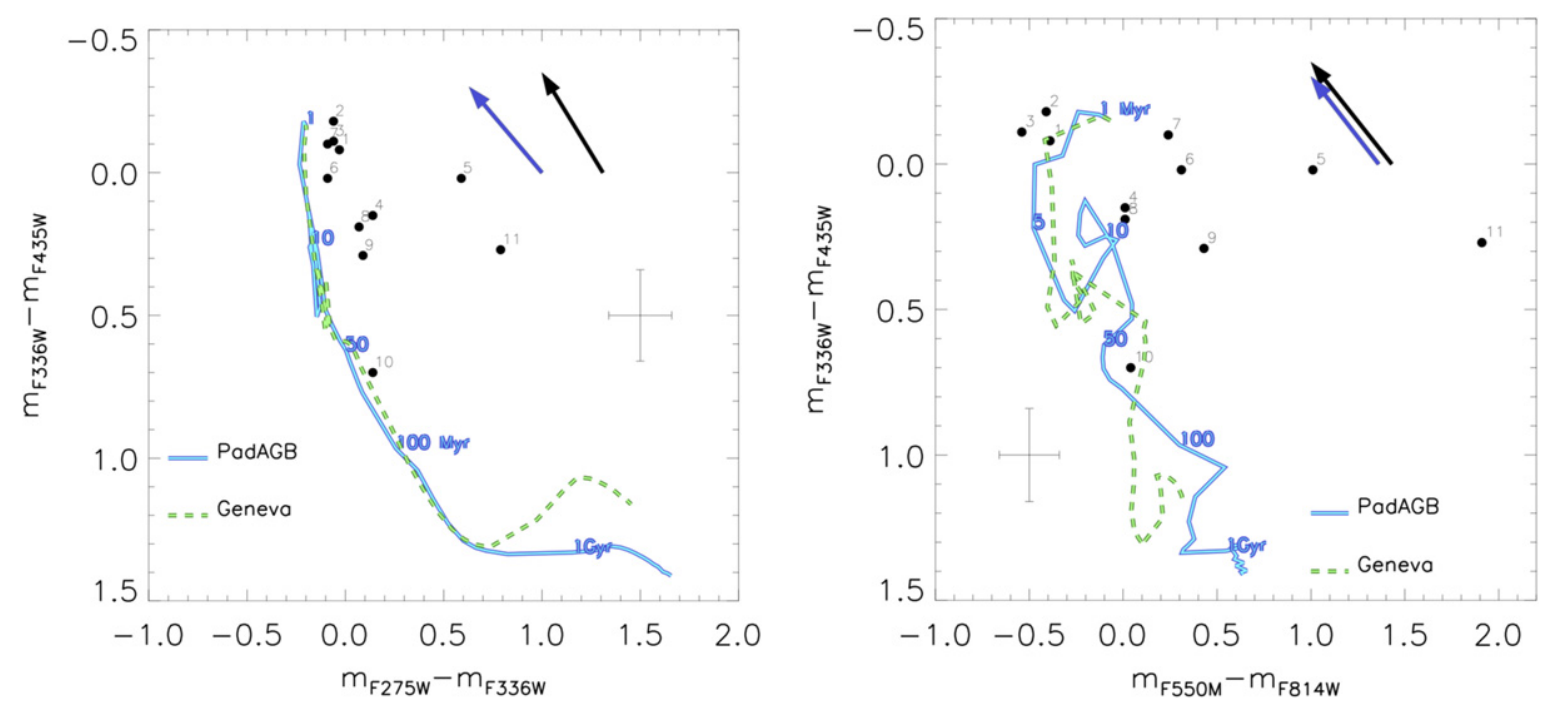

Figure 3. Selected color-color plots for the 11 star clusters shown in Figure 1. The U-B $\left(m_{F 336 \mathrm{~W}}-m_{F 435 \mathrm{~W}}\right)$ is shown as a function of both the NUV-U ( $m_{F 275 \mathrm{~W}-}$ $m_{F 336 \mathrm{~W}}$; left panel) and the V-I $\left(m_{F 550 \mathrm{M}^{-}} m_{F 814 \mathrm{~W}}\right.$; right panel). All magnitudes are on the Vega photometric scale. Typical photometric uncertainties are shown as thin crosses in the two panels. The location of synthetic colors for a range of ages, from $1 \mathrm{Myr}$ to $1 \mathrm{Gyr}$, is also shown for comparison, for both the Padova stellar evolutionary tracks with AGB treatment and the Geneva tracks (Section 4). Vectors showing the effect on the observed colors of a dust correction equivalent to a color excess $E(B-V)=0.3$ are reported for a starburst attenuation curve (black arrow) and for an LMC extinction curve (blue arrow).

to half the attenuation of the nebular gas (Calzetti et al. 1994; Kreckel et al. 2013). For the case of the starburst attenuation curve, the dust geometry is "built-in" into the functional form of the curve, and the differential attenuation between gas and stars is part of the way the curve itself was derived. We thus end up with seven different models for the dust attenuation: one attenuation curve and six extinction curves (three times two different ways of attenuating gas and stars). We generate the models in the color excess range $E(B-V)=0-3 \mathrm{mag}$, with step 0.01 .

We will see in the next section that cluster 11 cannot be easily explained by foreground extinction/attenuation only. For this case, we generate models in which the dust and stars/ gas are uniformly mixed together, according to the formula:

$$
\begin{aligned}
F(\lambda)_{\text {out }}= & F(\lambda)_{\text {model }}\left\{1-e^{[-0.921 E(B-V) k(\lambda)]}\right\} / \\
& {[0.921 E(B-V) k(\lambda)], }
\end{aligned}
$$

with the color excess $E(B-V)$ in the range 0-20 mag. Although the uniformly mixed geometry is likely to be an oversimplification of the complex environment surrounding cluster 11, it helps explain many of the properties of the star cluster. Throughout this paper, we will call "front-to-back optical depth" the quantity $A_{V}=3.1 E(B-V) k(V)$ from the mixed geometry.

The dust-attenuated SEDs are then convolved with the transmission curve of the filter plus the HST optics to produce synthetic luminosities, that are normalized to the default mass of Starburst99, $10^{6} M_{\odot}$.

We use $\chi^{2}$-minimization between the models and the data, taking into account the measurement uncertainties, to obtain the distribution of solutions and the reduced $\chi^{2}$ value for each. We then plot the distribution of solutions within the $99 \%$ significance level for the appropriate number of degrees of freedom, and select the best values and the uncertainty for the age, color excess, and mass of each star cluster based on the shape of the reduced $\chi^{2}$ probability distribution. We fit only the stellar continuum (medium/broad-band filter) photometry up to and not including the H-band. Both the J-band and the H-band can be heavily affected by the presence of small numbers of red supergiant stars (Cerviño \& Luridiana 2004; de Grijs et al. 2013; Gazak et al. 2013). In order to retain as much as possible of the wavelength baseline, we include the J-band in our fits, but exclude the H-band, and we only use it as a sanity check on our results. We use the hydrogen emission line intensities and the $\mathrm{H} \alpha \mathrm{EW}$ as a check on our solutions, by deriving an approximate age from the $\mathrm{H} \alpha \mathrm{EW}$ and a range of color excesses from the emission line ratios. We do not include the emission lines in the fit directly, since these can be affected by feedback effects from the star clusters (e.g., supernova explosions, which begin within the first $3 \mathrm{Myr}$, can eject gas from the cluster's surroundings and lead to an underestimate of the emission line intensity), especially for the massive clusters we are studying.

As presented in the next section, some of the star clusters have best fit ages around $1 \mathrm{Myr}$. This implies that pre-mainsequence stars could be present and contribute to the observed SEDs. Our models do not include pre-main-sequence stars, and this should be taken as a limitation to our approach.

We derive three solutions for the age, color excess, and mass of each star cluster from SED fitting. Two are based on the full wavelength coverage from $\sim 1500$ to $\sim 11000 \AA$ ( 7 data points $=3$ degrees of freedom, we average together the two measurements in $\mathrm{U}$ and the two measurements in $\mathrm{J}$, to produce one single data point at each wavelength), using Starburst99 and Yggdrasil models for the nebular-line-subtracted and unsubtracted photometry, respectively. The solutions from the comparison of the unsubtracted photometry with the Yggdrasil models are our reference values. We use the sets of solutions from the subtracted photometry plus Starburst 99 models as a comparison, in order to evaluate how well CLOUDY reproduces the conditions of the nebular gas in each star cluster. This is particularly important for the central clusters in NGC 5253, where the strong ionized gas emission can affect the measurements (e.g., by leaving residual emission in the 
Table 4

Physical Parameters of the Clusters Outside the Radio Nebula

\begin{tabular}{|c|c|c|c|c|c|c|}
\hline $\begin{array}{l}\text { Cluster } \\
(\#) \\
(1)\end{array}$ & $\begin{array}{r}\mathrm{Age}_{\text {SED }}{ }^{\mathrm{a}} \\
(\mathrm{Myr}) \\
(2)\end{array}$ & $\begin{array}{r}\text { Age }_{\text {SED-lines }}{ }^{\mathrm{a}} \\
(\mathrm{Myr}) \\
(3)\end{array}$ & $\begin{array}{r}\operatorname{Age}_{\mathrm{EW}(\mathrm{H} \alpha)}{ }^{\mathrm{b}} \\
(\mathrm{Myr}) \\
(4)\end{array}$ & $\begin{array}{r}\operatorname{Mass}_{\mathrm{SED}}{ }^{\mathrm{c}} \\
\left(10^{4} M_{\odot}\right) \\
(5)\end{array}$ & $\begin{array}{r}E(B-V)_{\mathrm{SED}}{ }^{\mathrm{d}} \\
(\mathrm{mag}) \\
(6)\end{array}$ & $\begin{array}{r}E(B-V)_{\text {lines }}{ }^{\mathrm{e}} \\
(\mathrm{mag}) \\
(7)\end{array}$ \\
\hline$\# 1$ & $\overline{5_{-2}^{+1}}$ & $5_{-1}^{+1}$ & $4.6-5.6$ & $1.05_{-0.22}^{+0.28}$ & $0.12_{-0.03}^{+0.03}$ & $\overline{0.12 \pm 0.19}$ \\
\hline$\# 2$ & $5_{-2}^{+1}$ & $5_{-2}^{+1}$ & $5.1-6.1$ & $0.91_{-0.22}^{+0.31}$ & $0.08_{-0.03}^{+0.03}$ & $0.15 \pm 0.19$ \\
\hline$\# 3$ & $5_{-0}^{+1}$ & $5_{-2}^{+1}$ & $7.5-10.1$ & $0.46_{-0.10}^{+0.11}$ & $0.04_{-0.02}^{+0.02}$ & $0.22 \pm 0.21$ \\
\hline$\# 4$ & $6_{-2}^{+0}$ & $5_{-1}^{+1}$ & $6.0-6.5$ & $1.62_{-0.48}^{+0.52}$ & $0.32_{-0.04}^{+0.04}$ & $0.22 \pm 0.19$ \\
\hline$\# 6$ & $10_{-1}^{+2}$ & $10_{-2}^{+8}$ & $>30$ & $3.24_{-0.94}^{+1.33}$ & $0.12_{-0.02}^{+0.04}$ & $\ldots$ \\
\hline$\# 7$ & $10_{-1}^{+3}$ & $10_{-2}^{+4}$ & $10-11$ & $1.15_{-0.56}^{+0.30}$ & $0.05_{-0.03}^{+0.04}$ & $0.37 \pm 0.22$ \\
\hline$\# 8$ & $15_{-3}^{+3}$ & $16_{-4}^{+4}$ & $>30$ & $2.88_{-0.84}^{+1.64}$ & $0.16_{-0.04}^{+0.04}$ & $1.92 \pm 1.22$ \\
\hline$\# 9$ & $10_{-1}^{+2}$ & $10_{-2}^{+4}$ & $11-16$ & $5.13_{-1.50}^{+2.12}$ & $0.26_{-0.04}^{+0.06}$ & $1.63 \pm 0.19$ \\
\hline$\# 10$ & $9_{-2}^{+5}$ & $12_{-4}^{+5}$ & $>16$ & $3.63_{-1.34}^{+3.22}$ & $0.26_{-0.04}^{+0.04}$ & $0.86 \pm 0.26$ \\
\hline
\end{tabular}

Notes.

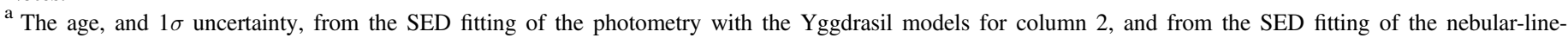
subtracted photometry with the Starburst99 models for column 3 .

$\mathrm{b}$ The age inferred from the $\operatorname{EW}(\mathrm{H} \alpha)$ listed in Table 3.

c The cluster mass as derived from the SED fitting with the Yggdrasil models.

d The color excess, with its $1 \sigma$ uncertainty, derived from SED fitting with the Yggdrasil models.

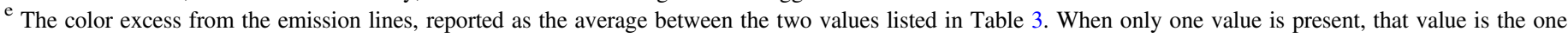
reported here.

stellar continuum bands). A third set of solutions is based on using only 5 bands (F275W, F336W, F435W, F550M, and $\mathrm{F} 814 \mathrm{~W}=1$ degree of freedom) for the best fits. This third set enables us to compare the solutions obtained from the more restricted wavelength range (which is the common situation for galaxies in the LEGUS and other projects) against those, possibly more secure, obtained from the more extended wavelength coverage.

\section{THE AGES, MASSES, AND EXTINCTIONS OF BRIGHT STAR CLUSTERS}

\subsection{Clusters outside the Radio Nebula}

Clusters 1-4 and 6-10 are located outside the radio nebula, although still within the starburst region. All except for cluster 4 have been investigated before by Calzetti et al. (1997), Tremonti et al. (2001), Harris et al. (2004), and de Grijs et al. (2013). All are younger than 15-20 Myr, as determined by those authors, using either lower resolution HST data, from the WFPC2, or UV spectroscopy, or a combination of ACS/HRC, WFPC2, and NICMOS data. Those earlier papers using broad and narrow-band photometry employ a more restricted wavelength range, and generally only one emission line $(\mathrm{H} \alpha)$. In our case, the availability of filters further in the UV (F125LP and F275W) provides better leverage for constraining ages of the star clusters from photometry, and the presence of multiple emission lines enables additional considerations on the physical conditions surrounding the clusters.

The best fit ages, masses, and color excesses, with their $1 \sigma$ uncertainties, are listed in Table 4 for these clusters. ${ }^{47}$ For each cluster, we generate separate files sorted by reduced $\chi^{2}$ values, and listing ages, color excesses, and masses for different combinations of stellar tracks (Geneva, Padova) and extinction/attenuation curves (MW, LMC, and SMC, both with and

\footnotetext{
47 The masses of all clusters would increase by about $60 \%$ if NGC 5253 were located at a distance of $4 \mathrm{Mpc}$, instead of our adopted $3.15 \mathrm{Mpc}$. Changing the stellar IMF from Kroupa to Salpeter also increases masses by a factor 1.6, for the same $0.1-120 M_{\odot}$ stellar range.
}

without differential treatment of lines and stellar continuum, and starburst curve). These files are used to determine both the best fits and the $99 \%$ confidence histograms, an example of which is given in Figure 4 for cluster 1. The histograms enable us to evaluate the uncertainties associated with each parameter, and these are the $1 \sigma$ uncertainties reported in Table 4 , but do not carry information on the best fits (i.e., on which of the 14 combinations of stellar tracks and extinction curves provides the best fit to the measured photometry). We infer the best fit values by extracting the model with the smallest $\chi^{2}$ value directly from the files, and the resulting synthetic SEDs and photometry are shown for all nine clusters in Figures 4 (top-left panel), 5, and 6 .

A few common characteristics emerge for all nine clusters from the exercise above. All are better fit by Padova stellar tracks, and, within the limit of validity of our foreground dust extinction assumptions, by the differential LMC or by the starburst attenuation curve. In this context, "better" means that the reduced $\chi^{2}$ is at least $50 \%$, and often more than a factor of 2 , smaller than for all other solutions. For ages $<6 \mathrm{Myr}$, the Padova tracks cluster around $5 \mathrm{Myr}$, while the Geneva tracks tend to cluster around $3 \mathrm{Myr}$ for the best-fit values. There is also a transition for the best fitting dust extinction/attenuation: younger clusters $(<6 \mathrm{Myr})$ prefer the differential LMC extinction, while older clusters prefer the starburst attenuation curve, which has the differential treatment of lines and stellar continuum "built in." Thus, differential extinction/attenuation is always required by the best fits solutions, i.e., emission lines are required to be more attenuated than the stellar continuum. In this case, we expect the color excess derived from line ratios to be larger than that derived for the stellar continuum from SED fitting. To test this, Table 4 lists side-by-side $E(B-V)$ values from SED fitting and from emission line ratios (columns 5 and 6 , respectively). The two sets of values are generally consistent with each other and, within the large error bars of the line-derived $E(B-V)$, we cannot exclude that the latter can be larger than the SED-derived $E(B-V)$. Indeed, Monreal-Ibero et al. (2010) finds evidence for differential extinction in 

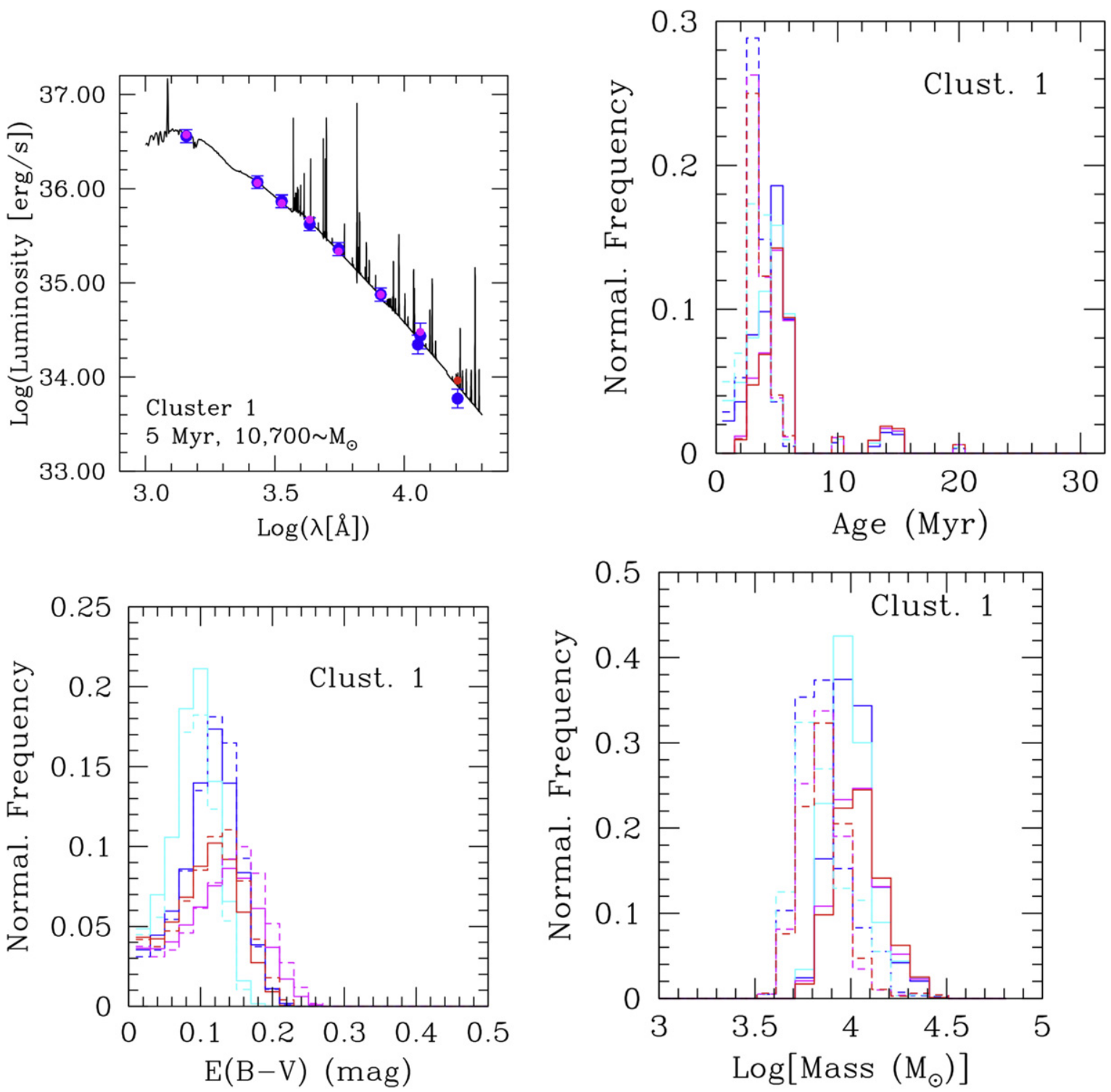

Figure 4. Best fit SED (top-left panel) and 99\% confidence histograms for the distribution of ages (top-right panel), color excess (bottom-left panel), and mass (bottom-right panel) for Cluster 1. In the best-fit SED plot, the blue points with error bars are the observed photometry, the magenta points are the synthetic photometry, and the black line is the Yggdrasil model, together with dust attenuation and mass normalization, that provides the best fit (smallest reduced $\chi^{2}$ value) to the observed photometry. The measured photometry in the H-band is not used in the fits, in order to avoid potential contamination by the stochastic presence of red supergiant stars; we show, however, the predicted photometric value (in red) from the best fit model. In the histograms, the continuous lines are for Padova stellar models and the dashed lines for Geneva stellar models. The colors indicate: differential LMC extinction curve (blue), differential SMC extinction curve (cyan), differential MW extinction curve (magenta), and starburst attenuation curve (red).

NGC 5253, with the stars being less attenuated than the gas by a factor 0.33 .

The main effect of differential extinction/attenuation between lines and continuum in the SED fits is to reduce the contribution of emission lines to the synthetic photometry in the broad/medium band filters, more than what is already accomplished by constructing models that assume only half of the ionized gas is in front of the clusters. A similar reduction effect can be obtained if the gas covering factor is lower than 0.5 ; indeed, the aperture correction for the $\mathrm{H} \alpha$ line is a factor over 2.5 larger than that for the underlying stellar continuum, suggesting a covering factor around 0.4. Furthermore, a decrease in the contribution of the metal lines (the major contributors to the broad band filters) can be accomplished by changing the ionization parameter in the CLOUDY models.
Thus, differential extinction/attenuation should not be considered a unique solution in this case.

For all clusters, we also show the NICMOS/NIC2/F160W photometry values predicted by the best-fit SEDs in Figures 4 (top-left panel), 5, and 6. In all nine cases, the prediction is within $2 \sigma$ of the observational value, lending further support to our results.

As a comparison, we report in Table 4 the best-fit ages and $1 \sigma$ uncertainties as obtained from fitting the photometry from nebular-line-subtracted images with Starburst99 population synthesis models. We use a method similar to the one used for the Yggdrasil models to derive ages and uncertainties, with the only change that we do not need to apply differential extinction, since the lines are no longer included in the SEDs (the nebular continuum is generally a much smaller 

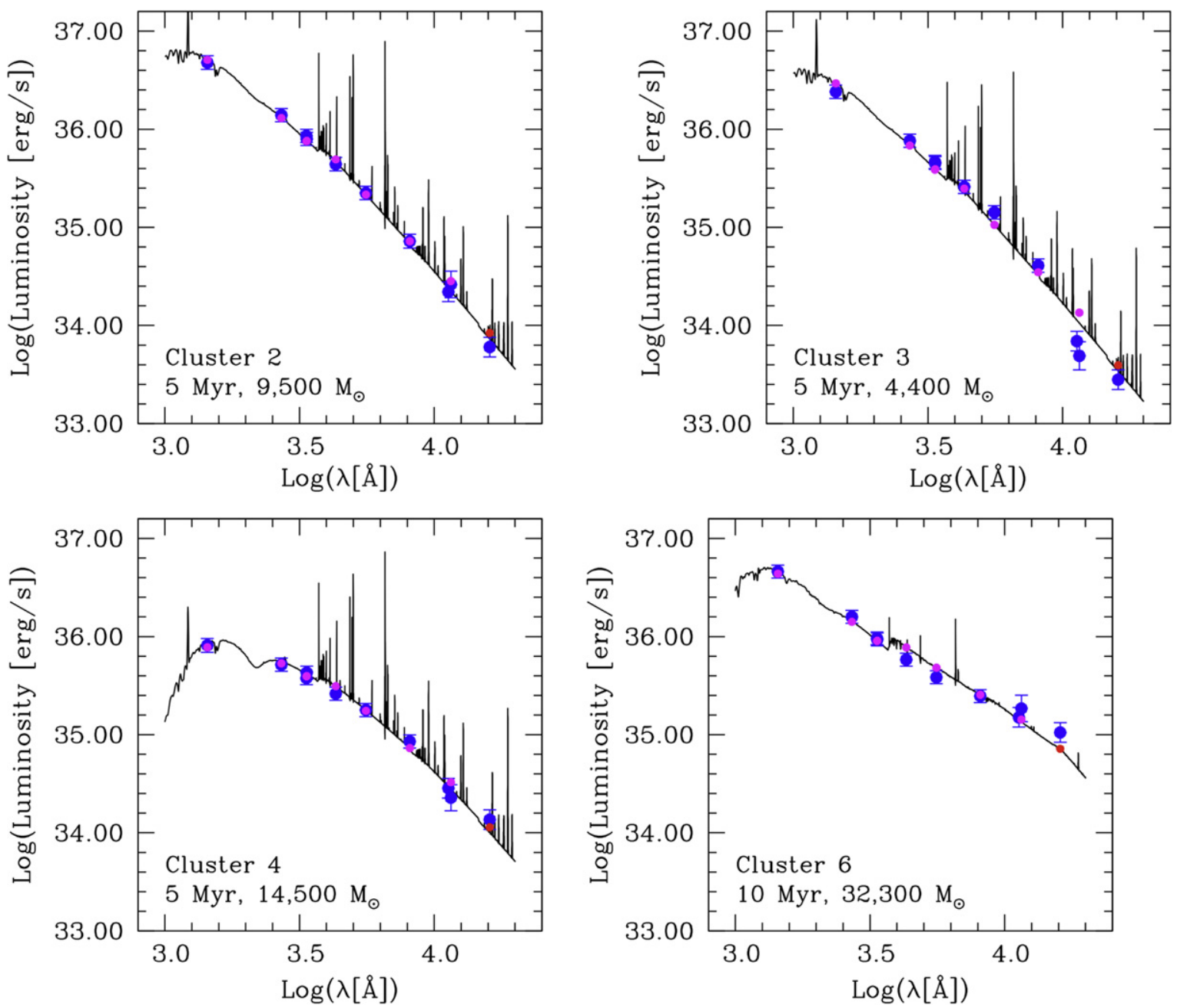

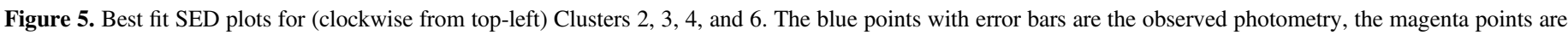

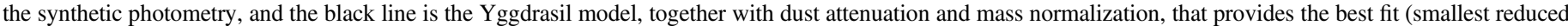

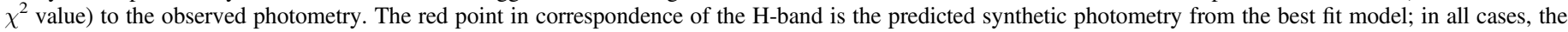
predicted photometry is within $2 \sigma$ of the observed photometry, although the H-band data are not used in the fitting.

contribution than the lines). As before, the younger clusters, 1 through 4, are better fit by an LMC extinction curve with Padova+AGB tracks, although the Geneva tracks give an almost as good best fit in all cases; the Padova and Geneva tracks yield a peak age of $5 \mathrm{Myr}$ and $3 \mathrm{Myr}$, respectively, which implies differences in the best fit masses of roughly a factor of 2 (with the masses from the Geneva tracks being the smaller of the two). Older clusters (6-10) are better fit by Padova stellar tracks with the starburst obscuration curve in the nebular-linesubtracted case, as well. The masses and color excesses are also in agreement between the fits performed on the photometry with and without nebular emission line: they are well within the $1 \sigma$ uncertainty for $E(B-V)$, and are within $70 \%$ of each other for the mass. The main exception is cluster 6 , with an estimated mass that is a little over two times larger for the nebular-linesubtracted photometry than for the unsubtracted photometry. This cluster shows a more marked tail toward older ages than other clusters, which accounts for the discrepancy in the most likely mass value.

The SED-derived ages are listed in Table 4 side-by-side with the ages inferred from the $\operatorname{EW}(\mathrm{H} \alpha)$. The latter age is a shorthand for checking whether the ionizing photon rates recovered through the emission line are consistent with those expected from the best-fit SED. For clusters $\sim 10$ Myr old and older, i.e., clusters 6-10, any agreement or discrepancy may be caused by uncertainties, since the $\mathrm{H} \alpha$ emission is expected to be low in this age range. It is, however, encouraging that, for the most part, there are no major discrepancies between the SED-derived and the $\operatorname{EW}(\mathrm{H} \alpha)$-derived ages for these clusters. For the younger clusters, 1, 2, and 4, the two ages are in good agreement. This result also suggests that ionizing photon leakage outside the $\mathrm{H}$ II regions and direct dust absorption of ionizing photons are not significant within/around these star clusters. For cluster 3 , the SED-derived age is $\sim 5 \mathrm{Myr}$, but the $\mathrm{EW}(\mathrm{H} \alpha)$ suggests values in excess of $7 \mathrm{Myr}$. The disagreement between the two age indicators can be ascribed to the discrepancy between the observed and the best-fit photometry in the V and I bands (top-left panel of Figure 5); the stellar continuum used to derive the $\operatorname{EW}(\mathrm{H} \alpha)$ is obtained from the interpolation between these two bands. The observed continuum values are higher than the predicted ones, possibly due to untreated uncertainties in the photometry and in the nebular emission, and yield an underestimated $\operatorname{EW}(\mathrm{H} \alpha)$. Within the uncertainties, no discrepancy is observed between the predicted and the measured, attenuation-corrected $\mathrm{H} \alpha$ luminosity (see Section 5.2). 

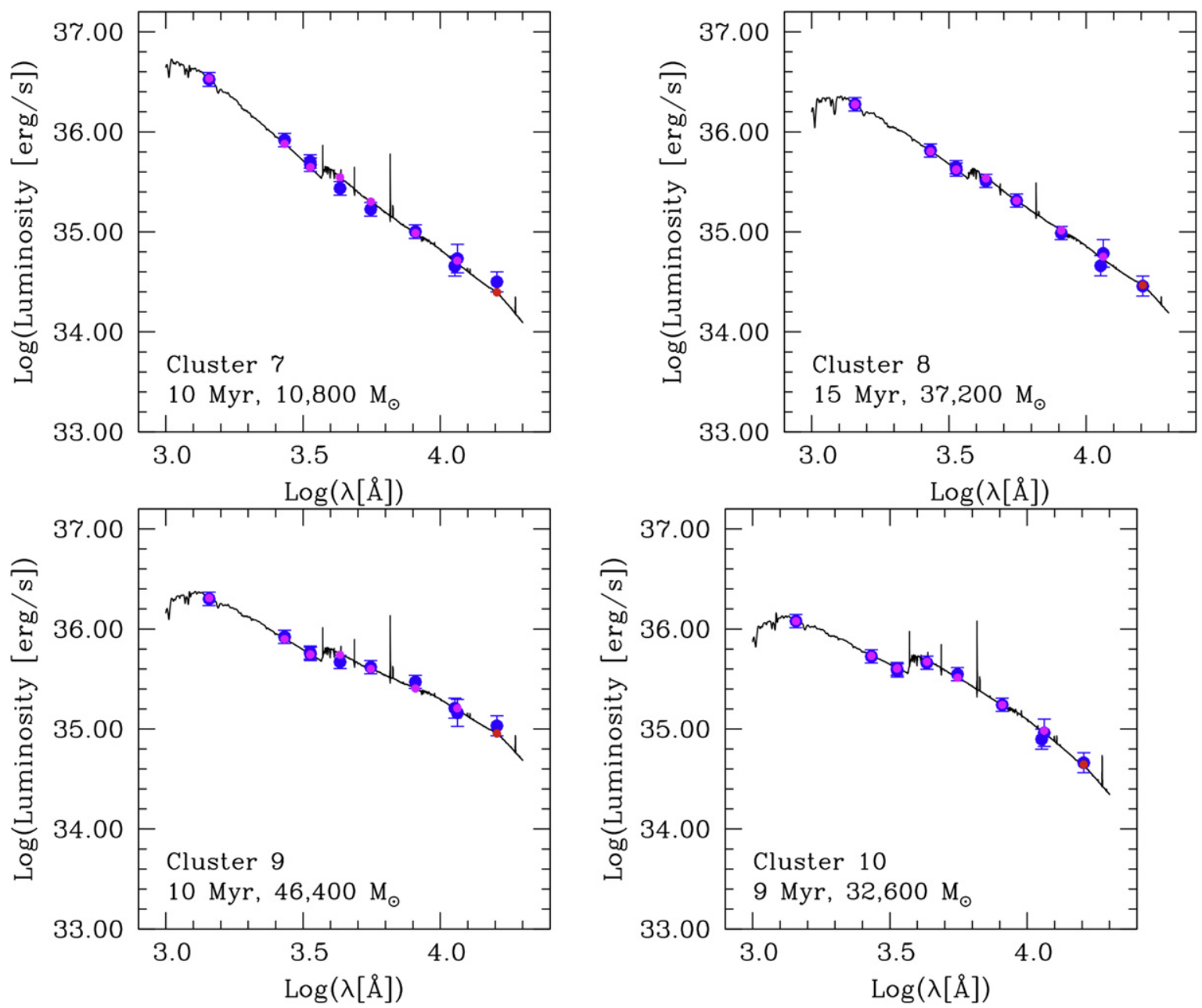

Figure 6. Same as in Figure 5, but for Clusters 7, 8, 9, and 10.

Cluster 3 is the one with the smallest mass among the nine analyzed so far, and, at a mean value around $4500 M_{\odot}$, may be showing some effects of stochastic sampling of the IMF (Cerviño \& Luridiana 2004; de Grijs et al. 2013; Gazak et al. 2013). For instance, stochastic effects may account for the low observed photometry in the near-IR relative to expectations from the best fit SED model (Figure 5): the observed SED is possibly deficient in red supergiants, which are bright, but rare, stars that become prominent near-IR contributors at approximately the age of cluster 3 (Popescu \& Hanson 2010; Anders et al. 2013). The low mass of cluster 3 requires further verification that we are dealing with a bona-fide star cluster, rather than a single, isolated star. From its attenuation-corrected SED, we infer that this cluster contains at least $10 \mathrm{O}$ stars, thus it is unlikely to be an isolated massive star.

Fitting five bands (NUV, U, B, V, I) recovers physical parameters that are close to those obtained with the seven bands fits for $7 / 9$ clusters. In all cases, the extinction/attenuation model needs to be specified a priori, in order to converge. We run tests using both the starburst curve and the LMC extinction curve with differential treatment of lines and continuum for the clusters younger than $6 \mathrm{Myr}$ and the starburst curve only for older star clusters. The goal is to check whether the starburst curve can be adopted in all cases. Figure 7 shows the results for Clusters 1 and 10, that are representative of the seven clusters with consistent solutions for seven and five band fits. Cluster 1 displays a preference for young ages independently of the extinction/attenuation model selected (either LMC or starburst), although the LMC yields better reduced $\chi^{2}$ values, by about $50 \%$. Cluster 10 shows the same double peak, with a range from $\sim 7$ to $15 \mathrm{Myr}$ in both cases.

The remaining two clusters, cluster 4 and cluster 6 , yield results that are not as robust as the other seven clusters, when using five band fits. The left panels of Figure 8 show the 99\% confidence histograms for cluster 4 , for the two cases of seven bands (red) and five bands fits (black). When five bands are used, the young age of cluster 4 is only marginally preferred for the Yggdrasil fits (top-left panel of Figure 8), i.e., when fitting broad/medium band photometry that include emission lines. However, when using Starburst 99 to fit photometric data from which emission lines have been subtracted, the preferred age for cluster 4 is markedly young, around $4-5 \mathrm{Myr}$, for both seven band and five band fits (bottom-left panel of Figure 8). Results are more complicated for cluster 6: fitting five bands yields an extremely young age $(\approx 1-2 \mathrm{Myr})$ when emission lines are included in the photometry (top-right panel of Figure 8), but marginally prefers the older age of $\sim 10 \mathrm{Myr}$ when emission lines are subtracted (bottom-right panel of Figure 8). The older age is preferred by the seven band photometry in both cases. For both cluster 4 and cluster 6 , removing emission lines from the photometric data yields better agreement in the recovered ages between the restricted 

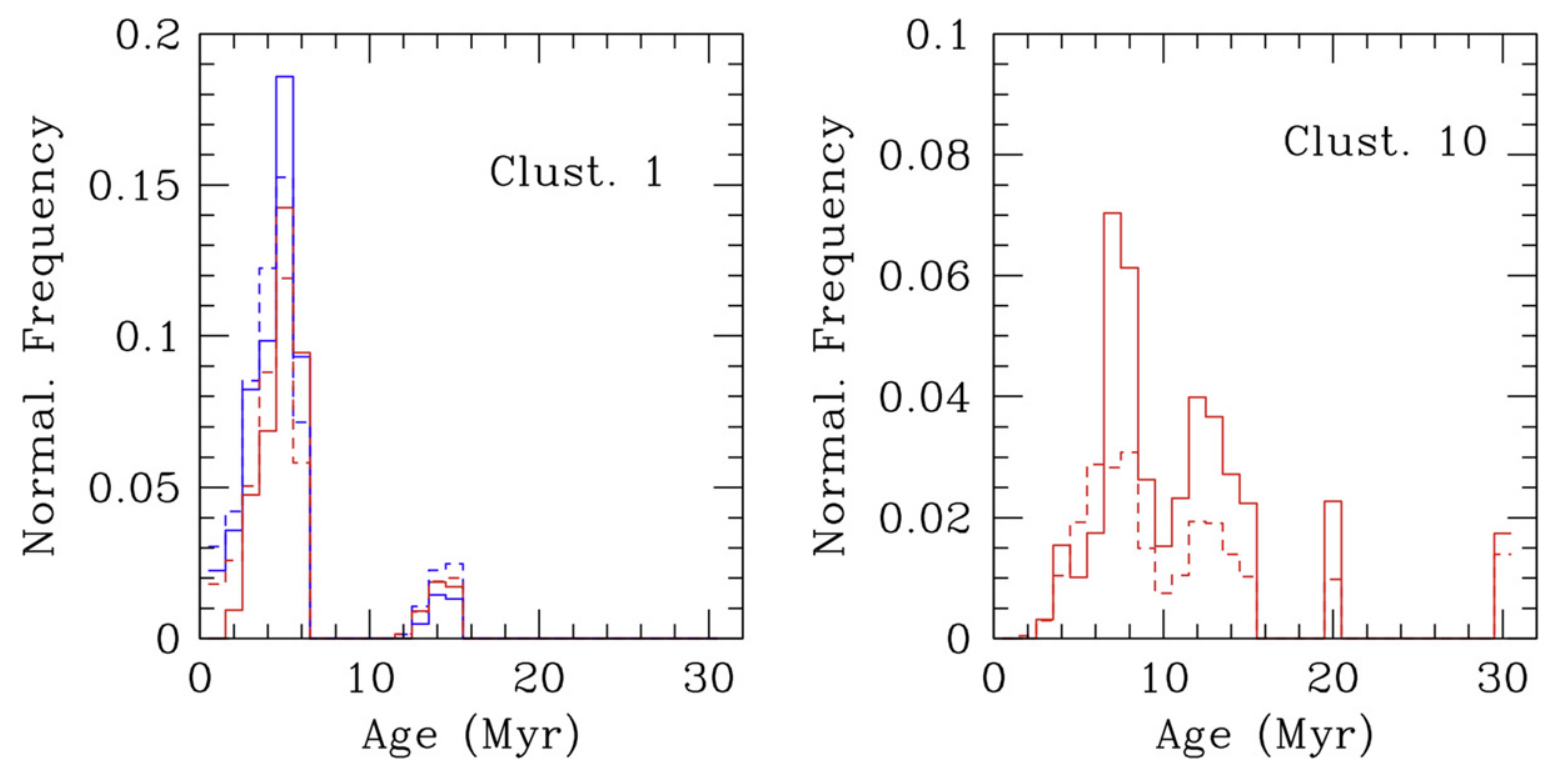

Figure 7. Confidence histograms for the distribution of ages for Cluster 1 (left) and Cluster 10 (right) for seven bands SED fits (continuous line) and 5 band fits (dash line). Both a differential LMC extinction curve (blue) and the starburst attenuation curve (red) are used for Cluster 1, while only the starburst curve is used for Cluster 10. For both cases, the starburst curve provides consistent results for both seven and five bands fits, also in the case of Cluster 1, which has a better solution with the differential LMC curve.

and the more extended wavelength ranges. This indicates that the method by which emission lines are included in Yggdrasil may not reflect reality in a small fraction of the sources. Verifying whether the observed effect is more general than indicated by our limited analysis will require a larger sample of star clusters.

\subsection{Clusters within the Radio Nebula}

The results from the previous section suggest that the SED fitting yields results that are internally consistent, and can be used to investigate the properties of clusters 5 and 11 . These clusters are highly attenuated by dust. The hydrogen line ratios reveal significant dust effects, with foreground values $A_{V} \sim$ $1.5 \mathrm{mag}$ and $4.7 \mathrm{mag}$ for clusters 5 and 11 , respectively.

The results of SED fitting for these two clusters are listed in Table 5, and shown in Figure 9. Both clusters are extremely young, with a best fit age of $1 \mathrm{Myr}$, which agrees with the age inferred from the $\mathrm{EW}(\mathrm{H} \alpha)$. Both are better represented by Geneva stellar tracks, and by the starburst attenuation curve for the foreground dust. As for the other clusters, we have verified that the best fit solution also reproduces the intensity and ratio of the line emission at $\mathrm{H} \alpha, \mathrm{P} \beta$, and $\mathrm{P} \alpha$. This is shown in Table 6, where we list, for all clusters, the intrinsic $\mathrm{H} \alpha$ luminosity, obtained from the attenuation-corrected measurements of Table 3, and the SED-predicted $\mathrm{H} \alpha$ luminosities, obtained from the models and the best-fit ages and masses of Tables 4 and 5 . Within the $1 \sigma$ uncertainties, there is general agreement between the two sets of values. Major disagreements are discussed below (cluster 5) and in Section 6.1 (cluster 9).

For cluster 5 the use of a simple dust geometry, i.e., foreground dust, is sufficient to recover an excellent fit for the SED, with a reduced $\chi^{2} \sim 1$. The color excess derived from the SED fitting agrees with the one derived from both line ratios: $\mathrm{H} \alpha / \mathrm{P} \beta$ and $\mathrm{H} \alpha / \mathrm{P} \alpha$. The emission lines are strong enough that, although the formal uncertainties are significant (Table 5), both ratios yield similar extinction values. Despite the goodness of fit, not all observational data points match the model SED: a significant deviation $(\sim 2 \sigma)$ is evident for the V-band (F550M), which may be due to difficulties in measuring the background surrounding the cluster. The observed photometry is below the model's value, which explains the unphysically large $\mathrm{EW}(\mathrm{H} \alpha)$ (Table 3).

The mass of cluster 5 derived from the SED fit is $\sim 7.5 \times 10^{4}$ $M_{\odot}$, confirming that this cluster is massive enough to drive the observed ionization. The $\mathrm{H} \alpha$ luminosity predicted for cluster 5 is $L(\mathrm{H} \alpha) \sim 5.7 \times 10^{39} \mathrm{erg} \mathrm{s}^{-1}$, to be compared with the attenuation-corrected measured value $L(\mathrm{H} \alpha)=2.8_{-0.7}^{+1.1}$ $\times 10^{39} \mathrm{erg} \mathrm{s}^{-1}$ (Table 6) and with the $\mathrm{H} \alpha$ luminosity inferred from the free-free measurement of Turner et al. (2000), $L(\mathrm{H} \alpha)$ $\approx 2-3 \times 10^{39} \mathrm{erg} \mathrm{s}^{-1}$ (the free-free measure likely provides an underestimate of the ionizing photon flux, since it is affected by self-absorption, see Meier et al. 2002). The discrepancy between the SED-predicted and the observed, attenuationcorrected $\mathrm{H} \alpha$ luminosity can be interpreted as due to either leakage of ionizing photons outside of the $\mathrm{H}$ II region or direct absorption of ionizing photons by dust. Leakage is supported by the presence of extended ionization around cluster 5 , which suggests that ionizing photons from this cluster reach further out than what we recover within our photometric apertures. We infer that $25 \%-50 \%$ of the ionizing photons produced by this star cluster leak out of the region. Direct absorption of ionizing photons by dust is also a potential mechanism in the dense environment surrounding cluster 5. However, we provide arguments both below and in Section 6.6 against a significant contribution from dust absorption of ionizing photons in this galaxy.

Cluster 11 requires a more complex approach to dust attenuation, in order to approximate the observed SED. A simple foreground dust model is unable to provide a reasonable fit to the observed SED, on account of the SED being mostly flat in $L(\lambda)$-versus- $\lambda$. A combination of a homogeneous duststar mixture and a foreground dust screen provide a better, albeit not perfect, fit (Figure 9). Flat or slightly blue SEDs in the presence of significant amounts of dust tend to require 

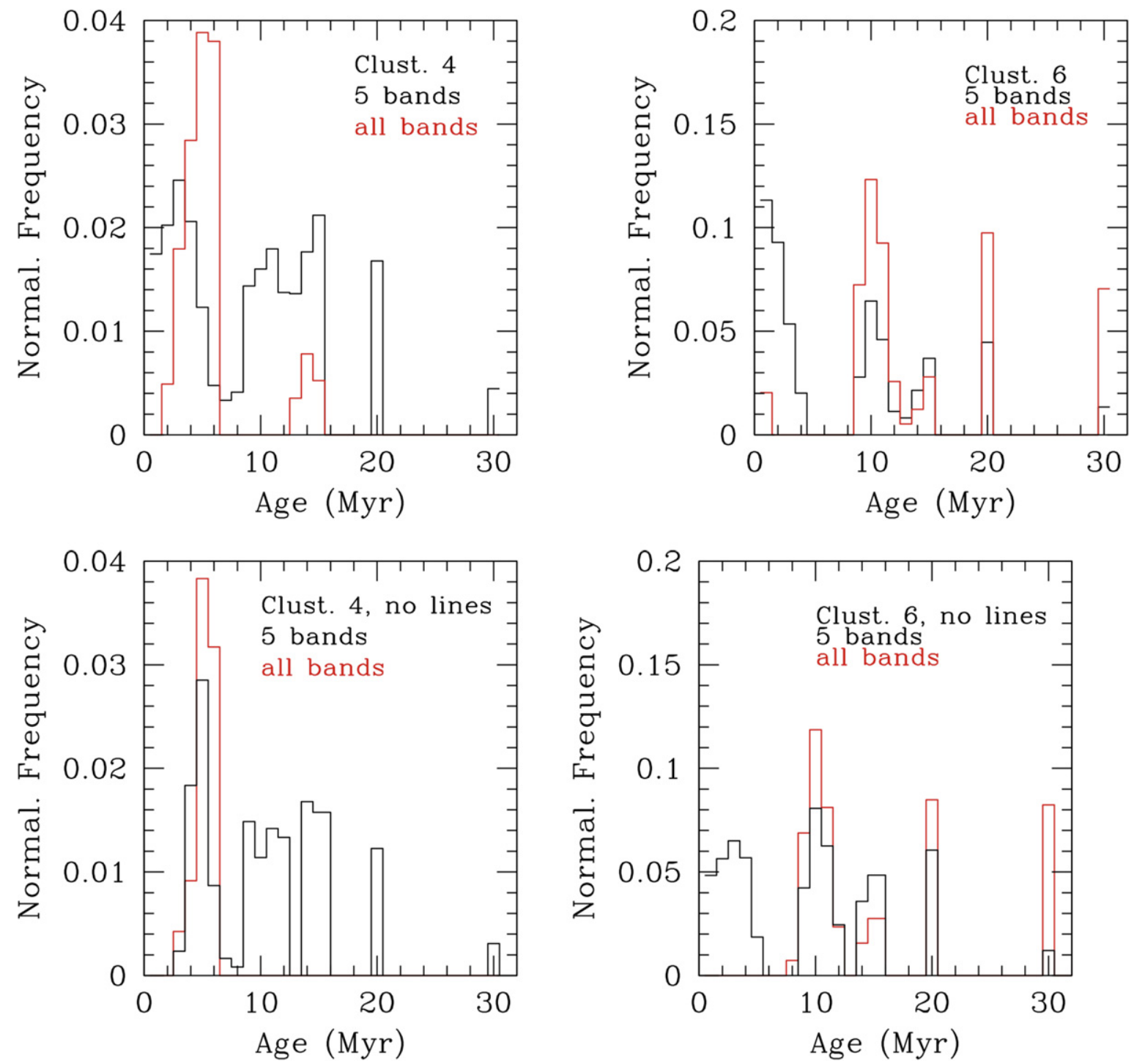

Figure 8. Histograms of the 99\% confidence level for the age distributions of Cluster 4 (left-hand side panels) and Cluster 6 (right-hand side panels), determined using all bands (red histograms) and only the five bands from the NUV to the I (black histograms). The top panels are for photometry that includes emission lines and fits performed with Yggdrasil models. The bottom panels are for photometry from which emission lines have been subtracted, and fits are performed with Starburst99 models. The agreement between all bands and five bands fits for the most likely age is higher when the emission lines are subtracted from the photometry.

models in which the dust is mixed with the stars. Foreground or shell models, either homogeneous or clumpy, will generally tend to produce too red SEDs relative to the data at blue wavelengths and too blue SEDs at red wavelengths (Calzetti 2001).

The major deviations between the data of cluster 11 and the model are in the $\mathrm{V}$ band and in the $\mathrm{J}$ and $\mathrm{H}$ bands. Like in cluster 5, the V-band observations are about $2 \sigma$ below the model, possibly an effect of background placement for the measurements. As in the other cluster, this accounts for the unphysically large $\mathrm{EW}(\mathrm{H} \alpha)$ measured for cluster 11 (Table 3). The other major deviation, in the near-IR bands, is in the opposite direction: the data are more luminous, by slightly more than $2 \sigma$, than the model expectation. A discussion of this deviation is deferred to Section 6.3.

Taken at face value, cluster 11 is the most massive among those analyzed here, with a mass $M \sim 2.5 \times 10^{5} M_{\odot}$. The component of dust that is mixed with the stars in the cluster has a front-to-back optical depth of $A_{V} \sim 49 \mathrm{mag}$ and follows the
Milky Way extinction curve; this curve yields a reduced $\chi^{2}$ that is at least two times better than any other of the extinction curves tested in this paper. The foreground dust component obeys the starburst attenuation curve, with $A_{V} \sim 1.9 \mathrm{mag}$. Cluster 11 is therefore associated with about 50 mag of optical depth in dust, although the mixed geometry allows for some of the UV-optical radiation to shine through. This optical depth is larger than what was determined by other authors. AlonsoHerrero et al. (2004) derive $A_{V} \sim 17$ mag for this cluster, but they only assume a foreground dust screen, which provides a lower limit to the actual dust optical depth of a region. MartinHernandez et al. (2005) derive a value of $A_{V} \sim 14-17 \mathrm{mag}$, from the $9.7 \mu \mathrm{m}$ silicate absorption feature; however, the relatively large slit they use for their spectroscopic observations $(1 ! 2 \sim 18 \mathrm{pc})$ is likely to have sampled regions of different optical depth, thus weighting the final result toward less extreme values of $A_{V}$. Calzetti et al. (1997) derive a larger range, $A_{V} \sim 9-35 \mathrm{mag}$, the upper bound of which is more consistent with the value derived here. 
Table 5

Physical Parameters of the Clusters within the Radio Nebula

\begin{tabular}{|c|c|c|c|c|c|c|}
\hline $\begin{array}{l}\text { Cluster } \\
(\#) \\
(1)\end{array}$ & $\begin{array}{r}\text { Age }_{\text {SED }}{ }^{a} \\
(\mathrm{Myr}) \\
(2)\end{array}$ & $\begin{array}{r}\operatorname{Age}_{\mathrm{EW}(\mathrm{H} \alpha)} \mathrm{b} \\
(\mathrm{Myr}) \\
(3)\end{array}$ & $\begin{array}{r}\operatorname{Mass}_{\mathrm{SED}} \mathrm{c} \\
\left(10^{4} M_{\odot}\right) \\
(4)\end{array}$ & $\begin{array}{r}E(B-V)_{\mathrm{SED}, \operatorname{mix}}^{\mathrm{d}} \\
(\mathrm{mag}) \\
(5)\end{array}$ & $\begin{array}{r}E(B-V)_{\mathrm{SED}, \text { fore }}^{\mathrm{d}} \\
(\mathrm{mag}) \\
(6)\end{array}$ & $\begin{array}{r}\begin{array}{r}(B-V)_{\text {lines }}^{\mathrm{e}} \\
(\mathrm{mag}) \\
(7)\end{array}\end{array}$ \\
\hline$\overline{\# 5}$ & $1_{-1}^{+1}$ & $<1$ & $7.46_{-0.27}^{+0.20}$ & $\cdots$ & $0.46_{-0.04}^{+0.04}$ & $0.48 \pm 0.19$ \\
\hline
\end{tabular}

Notes.

a The age, and $1 \sigma$ uncertainty, from the SED fitting of the photometry.

b The age inferred from the $\mathrm{EW}(\mathrm{H} \alpha)$ listed in Table 3.

${ }^{c}$ The cluster mass as derived from the SED fitting.

${ }^{\mathrm{d}}$ The color excess, with its $1 \sigma$ uncertainty, derived from SED fitting. The two values of $E(B-V)$ are for the mixed dust-stars and foreground dust cases. Cluster 11 requires both dust geometries to be present in order to account for the shape and luminosity of its observed SED.

${ }^{\mathrm{e}}$ The color excess from the emission lines, reported as the average between the two values listed in Table 3 .
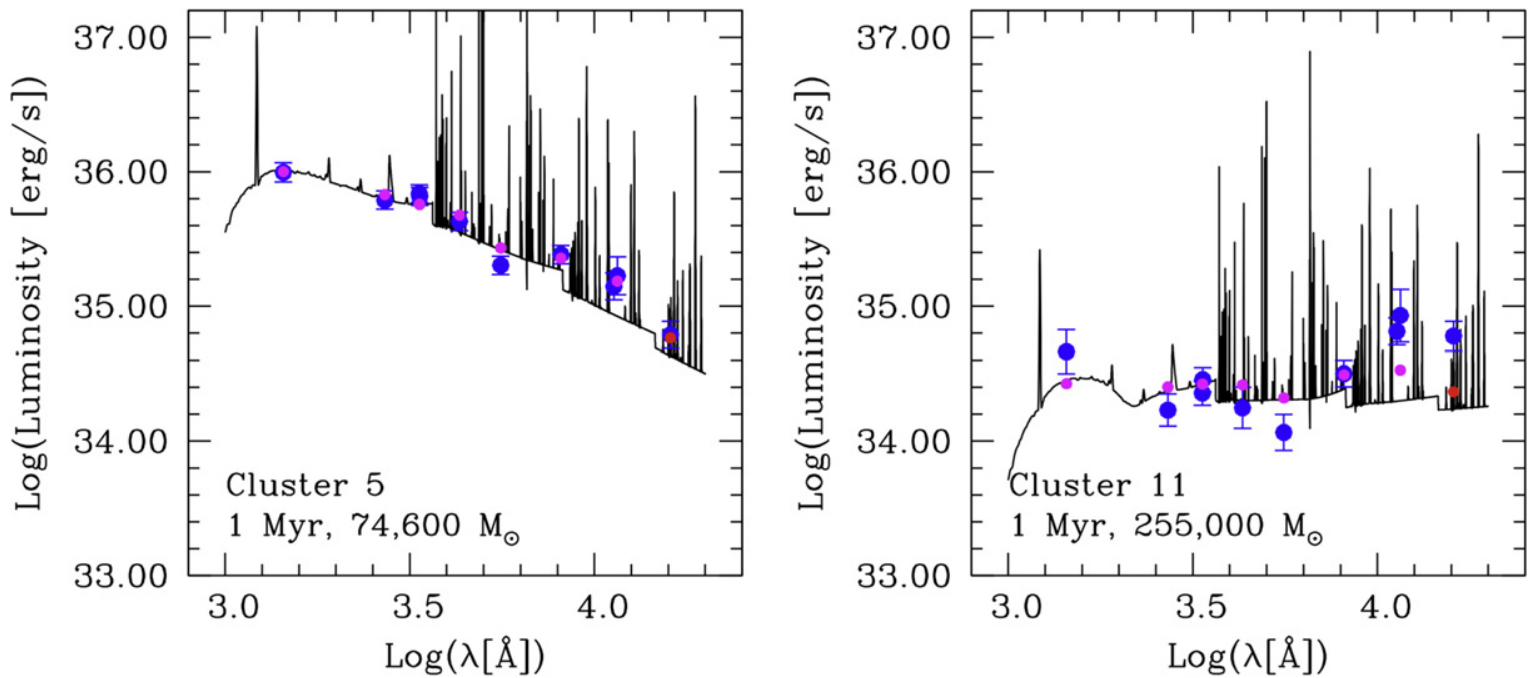

Figure 9. Best fit SEDs for Clusters 5 (left panel) and 11 (right panel). As in Figure 2, the blue points with error bars are the observed photometry, the magenta points are the synthetic photometry, and the black line is the Yggdrasil model, together with dust attenuation and mass normalization, that provides the best fit (smallest reduced $\chi^{2}$ value) to the observed photometry. For Cluster 5, only foreground dust is required to achieve a reduced $\chi^{2} \sim 1$. For Cluster 11 , a combination of both mixed dust, with a total dust column of $A_{V} \sim 48.7 \mathrm{mag}$, and foreground dust, with $A_{V} \sim 1.9 \mathrm{mag}$, are needed to approximate the observed SED.

From the best-fit model SED, the predicted $\mathrm{H} \alpha$ luminosity of cluster 11 is $L(\mathrm{H} \alpha) \sim 2 \times 10^{40} \mathrm{erg} \mathrm{s}^{-1}$, which is comparable to the measured, attenuation-corrected $L(\mathrm{H} \alpha) \sim 1.8_{-0.5}^{+0.7}$ $\times 10^{40} \mathrm{erg} \mathrm{s}^{-1}$ (Table 6). As a reminder, the full dust model of combined mixed and foreground dust is applied to all three emission lines, $\mathrm{H} \alpha, \mathrm{P} \beta$, and $\mathrm{P} \alpha$, to derive internally consistent values for their luminosities. The free-free emission within $4 \mathrm{pc}$ of the radio peak corresponds to an intrinsic $\mathrm{H} \alpha$ luminosity $L(\mathrm{H} \alpha) \sim 1.6-2.0 \times 10^{40} \mathrm{erg} \mathrm{s}^{-1}$ (from the $7 \mathrm{~mm}$ measurement of Turner $\&$ Beck 2004). The agreement between the measured, attenuation-corrected $\mathrm{H} \alpha$ luminosity and the SED-predicted luminosity provides independent confirmation of the accuracy of our results for cluster 11. Within the uncertainty of the measurements, the mass and age of cluster 11 are accurate, as are the dust content and geometry; there are no major parts of cluster 11 that are so deeply buried in dust to be unrecoverable by our approach. It also suggests that, within the uncertainties, direct absorption of ionizing photons by dust does not appear to be a dominant mechanism at work in the area where cluster 11 resides, despite the large dust optical depth.

Because of the red SED of cluster 11, a secondary best-fit solution is provided by a $\sim 100 \mathrm{Myr}$ old star cluster, with a mass $M \sim 3.5 \times 10^{5} M_{\odot}$, also mixed with dust with optical depth $A_{V} \sim 42 \mathrm{mag}$. We reject this solution on account of the strong nebular line emission in coincidence of the location of cluster 11. However, the uncertainties in both line emission and stellar continuum measurements allow the co-existence of two populations at the location of cluster 11: a $\sim 2 \times 10^{5} M_{\odot}, 1 \mathrm{Myr}$ old population together with a $\sim 1 \times 10^{5} M_{\odot}, 100 \mathrm{Myr}$ old population. This solution would push against the tolerance of our error bars, but is not formally excluded by the data.

Other sources of uncertainty for the best-fit solutions of cluster 11 include the requirement that emission line intensities and ratios be consistent with the model for the stellar continuum. If we allow the stellar continuum to be modeled independently of the emission lines, we obtain a larger range of degeneracies as a consequence of the larger number of degrees of freedom. For instance, in the absence of constraints from the emission lines, the dust-star mixed model produces a linear correlation between mass and front-to-back optical depth for $A_{V}>15$ mag: by doubling the total attenuation, one can double the cluster mass recovered with virtually unchanged $\chi^{2}$ values. 
Table 6

Intrinsic and Predicted $\mathrm{H} \alpha$ Luminosities

\begin{tabular}{lrr}
\hline \hline $\begin{array}{l}\text { Cluster } \\
(\#)\end{array}$ & $\begin{array}{r}\log \left[L(\mathrm{H} \alpha)_{\text {intrinsic }}\right]^{\mathrm{a}} \\
\left(\mathrm{erg} \mathrm{s}^{-1}\right)\end{array}$ & $\begin{array}{r}\log \left[L(\mathrm{H} \alpha)_{\text {predicted }}\right]^{\mathrm{b}} \\
\left(\mathrm{erg} \mathrm{s}^{-1}\right)\end{array}$ \\
\hline (1) & $(2)$ & $(3)$ \\
$\# 1$ & $38.38_{-0.12}^{+0.18}$ & $38.13_{-0.31}^{+0.39}$ \\
$\# 2$ & $38.21_{-0.15}^{+0.19}$ & $38.07_{-0.31}^{+0.72}$ \\
$\# 3$ & $37.62_{-0.21}^{+0.21}$ & $37.77_{-0.30}^{+0.72}$ \\
$\# 4$ & $37.85_{-0.19}^{+0.19}$ & $37.99_{-0.19}^{+0.73}$ \\
$\# 5$ & $39.45_{-0.12}^{+0.15}$ & $39.76_{-0.04}^{+0.04}$ \\
$\# 6$ & $<35.36$ & $37.26_{-1.16}^{+0.45}$ \\
$\# 7$ & $37.15_{-0.22}^{+0.22}$ & $36.81_{-0.68}^{+0.45}$ \\
$\# 8$ & $<37.31$ & $36.38_{-0.31}^{+0.41}$ \\
$\# 9^{c}$ & $38.62_{-0.19}^{+0.19}$ & $37.46_{-0.92}^{+0.46}$ \\
$\# 10$ & $36.81_{-0.26}^{+0.26}$ & $37.56_{-1.06}^{+0.49}$ \\
$\# 11$ & $40.25_{-0.13}^{+0.15}$ & $40.29_{-0.08}^{+0.10}$ \\
\hline
\end{tabular}

Notes.

${ }^{\mathrm{a}}$ The intrinsic $\mathrm{H} \alpha$ luminosity of each cluster, in $\log$ scale, including $1 \sigma$ uncertainties, derived from the measurements listed in Table 3, corrected for dust attenuation using Equation (1) and the color excess values listed in Tables 4 and 5. For cluster 11, both Equations (1) and (2) are used to remove the effects of dust attenuation.

b The predicted $\mathrm{H} \alpha$ luminosity, in log scale, from the best-fit ages and masses of each cluster, including uncertainties (Tables 4 and 5). Except for clusters 5 and 11 , the main contributor to the uncertainty in the predicted $L(\mathrm{H} \alpha)$ is the uncertainty in the best-fit age. For clusters 5 and 11, the main contributor to the overall uncertainty in $L(\mathrm{H} \alpha)$ is the uncertainty in the best-fit mass, since the ionizing photon rate is fairly constant for ages $\lesssim 2.5 \mathrm{Myr}$. The $\mathrm{H} \alpha$ luminosities are related to the ionizing photon rate $Q\left(\mathrm{H}^{o}\right)$ via: $L(\mathrm{H} \alpha)\left[\operatorname{erg~s}^{-1}\right]=1.37 \times 10^{-12}$ $Q\left(\mathrm{H}^{o}\right)\left[\mathrm{s}^{-1}\right]$ (Leitherer et al. 1999; Calzetti 2013).

${ }^{\mathrm{c}}$ The intrinsic $\mathrm{H} \alpha$ luminosity of cluster 9 is likely over predicted, by about an order of magnitude, due to the presence of a highly dust-attenuated, younger interloper (see discussion in Section 6.1). If using the color excess derived from the SED fits, rather than the line ratios (Table 4), the intrinsic $\mathrm{H} \alpha$ luminosity of cluster 9 decreases to $\log \left[L(\mathrm{H} \alpha)_{\text {intrinsic }}\right]=37.23_{-0.12}^{+0.13}$, in better agreement with the predicted $\mathrm{H} \alpha$ luminosity.

\subsection{Limitations in the Population Synthesis Models}

There are several properties of massive stars that are not currently included in the population synthesis models used in the present analysis. These are: stellar rotation, binary stars, and VMSs. Most of these properties are still under investigation, and their inclusion in models is either in the early stages (e.g., rotation and binaries) or non existent. We provide here a brief summary of the effects we expect each to have on our results.

A stellar population containing rotating massive stars will appear younger than a non-rotating counterpart, with an increase in the ionizing flux that can be as much as a factor two-five higher (Leitherer et al. 2014). Applied to the star clusters in our sample, such models are expected to yield older best-fit ages than what we derive or, alternatively, larger ionizing fluxes at fixed age.

Young massive stars are predominantly found in close binaries, and can interact (e.g., Sana et al. 2012). The products of such interactions include massive blue stragglers formed through mass transfer and mergers, which may be common (de Mink et al. 2014), and appear at the upper tail end of the stellar IMF (Schneider et al. 2014, 2015). The effects on young star clusters include apparent rejuvenation and age spreads (Eldridge \& Stanway 2009). Our data do not include enough
Table 7

Comparison with the Literature

\begin{tabular}{lrrrrr}
\hline \hline $\begin{array}{l}\text { Cluster } \\
\text { \#) }\end{array}$ & $\begin{array}{r}\mathrm{Age}^{\mathrm{a}} \\
(\mathrm{Myr})\end{array}$ & $\begin{array}{r}\mathrm{Age}_{C 97}{ }^{\mathrm{b}} \\
(\mathrm{Myr})\end{array}$ & $\begin{array}{r}\mathrm{Age}_{T 01}{ }^{\mathrm{c}} \\
(\mathrm{Myr})\end{array}$ & $\begin{array}{r}\mathrm{Age}_{\mathrm{H} 04}{ }^{\mathrm{d}} \\
(\mathrm{Myr})\end{array}$ & $\begin{array}{r}\mathrm{Age}_{d G 13} \mathrm{e} \\
(\mathrm{Myr})\end{array}$ \\
\hline$\# 1$ & $(2)$ & $(3)$ & $(4)$ & $(5)$ & $(6)$ \\
$\# 2$ & $5_{-2}^{+1}$ & $2.5-4.4$ & $\ldots$ & $1-5$ & $\ldots$ \\
$\# 3$ & $5_{-2}^{+1}$ & $2.5-4.4$ & $\ldots$ & $1-5$ & $\ldots$ \\
$\# 4$ & $5_{-2}^{+4}$ & $\ldots$ & $\ldots$ & $3-5$ & $\ldots$ \\
$\# 5$ & $6_{-2}^{+1}$ & $\ldots$ & $\ldots$ & $\ldots$ & $\ldots$ \\
$\# 6$ & $1_{-1}^{+1}$ & $<3$ & $2_{-0.8}^{+0.7}$ & $1-3$ & $\ldots$ \\
$\# 7$ & $10_{-2}^{+8}$ & $8-12$ & $\ldots$ & $10-11$ & $12-16$ \\
$\# 8$ & $10_{-2}^{+4}$ & $\ldots$ & $\ldots$ & 6 & $\ldots$ \\
$\# 9$ & $15_{-3}^{+4}$ & $10-17$ & $\ldots$ & $9-14$ & $12-16$ \\
$\# 11$ & $10_{-2}^{+5}$ & $30-50$ & $3_{-0.9}^{+0.9}$ & $11-14$ & $6-8$ \\
\hline 10 & $9_{-2}^{+7}$ & $50-60$ & $8_{-0.9}^{+2.5}$ & $8-15$ & $\ldots$ \\
\hline & $1_{-1}^{+1}$ & $\ldots$ & $\ldots$ & $\ldots$ & $\ldots$ \\
\hline
\end{tabular}

Notes.

a The ages, and $1 \sigma$ uncertainty, of the clusters in this work from the SED fitting and the $\operatorname{EW}(\mathrm{H} \alpha)$ constraints combined together.

b The ages derived by Calzetti et al. (1994).

c The ages derived by Tremonti et al. (2001), using UV spectroscopy from the HST/STIS instrument.

d The ages derived by Harris et al. (2004).

e The ages derived by de Grijs et al. (2013).

information to infer whether either of those effects may be present, but we cannot exclude them either.

The birth mass of stars is limited to $\sim 120-150 M_{\odot}$ in virtually all population synthesis models, but there is mounting evidence that stars as massive as $300 M_{\odot}$ are present in nearby young star clusters (Crowther et al. 2010). There is still debate on whether these VMSs are the result of birth conditions or of mergers, and stellar tracks are being produced in order to further investigate this issue (Yusof et al. 2013; Koehler et al. 2015). The youngest among our clusters, clusters 5 and 11 , are also sufficiently massive that they may contain VMSs. Presence of VMSs increases the ionizing photon flux from a cluster. If the VMSs contributions to clusters 5 and 11 were similar to those found in the LMC cluster R136, the ionizing photon flux could be 50\%-100\% larger than the one currently predicted (Crowther et al. 2010; Doran et al. 2013).

One final limitation of our model fitting is that the youngest synthetic population we consider is $1 \mathrm{Myr}$ old, in line with our photometric uncertainties which yield a best accuracy of $\sim 1 \mathrm{Myr}$ at those young ages. The formal solutions for clusters 5 and 11 do not exclude that they could be younger than $1 \mathrm{Myr}$.

\section{DISCUSSION}

\subsection{Age Comparisons with the Literature}

The physical parameters of age and mass have been derived before by several authors for most of the star clusters in this study (Calzetti et al. 1997; Tremonti et al. 2001; Harris et al. 2004; de Grijs et al. 2013). We compare our derived ages with those previous derivations in Table 7 . We do not compare masses, as these are somewhat degenerate with ages and depend on additional assumptions such as the stellar IMF and the adopted distance for NGC 5253. There is general agreement between the ages derived by all authors, with a few exceptions.

The most notable discrepancies are present for cluster 9, with age estimates that range from $3 \mathrm{Myr}$ (Tremonti et al. 2001) to 
30-50 Myr (Calzetti et al. 1997). The old age derived by Calzetti et al. (1997) is an effect of the limited number of broad bands available to those authors, since they only used NUV (centered at $\sim 2250 \AA$ ),, $\mathrm{V}$, and I; in particular, the absence of a filter close to the age-sensitive U-band limits the ability to attribute ages to star clusters (e.g., Lee et al. 2005). The younger age derived by Tremonti et al. (2001) is far more puzzling. These authors use UV spectroscopy for deriving cluster ages, leveraging the information from the photospheric lines, while all other authors derive their age estimates from colors or SED fitting. The UV spectrum of cluster 9 shows the presence of P-Cygni profiles for the lines of $[\mathrm{N} \mathrm{v}](\lambda 1240 \AA)$ and $[\mathrm{C} \operatorname{Iv}](\lambda 1550 \mathrm{~A})$, a clear sign for the presence of early O-stars and ages $\lesssim 5 \mathrm{Myr}$. However, the long slit of the HST/ STIS only "grazes" the outskirts of cluster 9 , with only $1 / 30$ th of the light from this cluster captured by the UV spectrum (Tremonti et al. 2001). Possibly, the UV spectrum is targeting a smaller cluster in the periphery of cluster 9 . The presence of one or more interlopers is supported by the anomalously large (for its age and location) color excess from emission lines for cluster 9 (Table 3); these large values for $E(B-V)$ are usually found in correspondence of much younger star clusters. Despite the large attenuation value, the emission lines in correspondence of the cluster are still weak for its mass (Table 6), and there is no evidence for additional ionized gas in the form of shells or arcs in the region; this further supports the older age, $\approx 10 \mathrm{Myr}$, for cluster 9 .

For cluster 10, the main discrepancy is given by the age reported by Calzetti et al. (1997), 50-60 Myr, while our determination and those from other authors suggest an age around 8-15 Myr. As in cluster 9, the discrepancy is due to the limited number of photometric bands available to Calzetti et al. (1997). An early suggestion that clusters 9 and 10 could be younger than the 30-60 Myr age inferred by Calzetti et al. (1997) came from Strickland \& Stevens (1999), based on the measured X-ray sizes and luminosities of the super bubbles in NGC 5253.

The young ages of the dusty clusters 5 and 11 have been known for quite some time (e.g., Meurer et al. 1995; Calzetti et al. 1997; Schaerer et al. 1997), but the realization that the region hosts two separate clusters instead of a single one is more recent (e.g., Alonso-Herrero et al. 2004). Alonso-Herrero et al. (2004), specifically, favors the presence of two star clusters over other interpretations, such as that cluster 5 may be a reflection nebula generated by cluster 11 (Turner \& Beck 2004). Our analysis also favors the interpretation of two separate star clusters. The morphology of cluster 5 is consistent with that of a compact star cluster: a slightly resolved $(\sim 0$ !" 1 corresponding to a deconvolved size of $\sim 1.2 \mathrm{pc}$ ), symmetric, and centrally concentrated source, similar to other clusters in the area, including those in the same $\sim 1^{\prime \prime}$ region as cluster 5 . At least an additional 6-7 fainter star cluster candidates are visible in the $\sim 0$ " 6 area surrounding cluster 5 , suggesting that this one is the most prominent one in an association of stars and/or star clusters.

Both clusters have been characterized as having ages $\sim 3-3.4$ Myr by Alonso-Herrero et al. (2004), based on HST near-IR imaging and ground-based spectroscopy; the inferred masses are $\sim 6.6 \times 10^{4} M_{\odot}$ for cluster 5 and $\sim 3.9 \times 10^{5}-$ $1.3 \times 10^{6} M_{\odot}$ for cluster 11 , when reported to our adopted distance and stellar IMF. For cluster 5, the difference between our mass and the mass derived by Alonso-Herrero et al. (2004) can be entirely attributed to differences in the derived ages and extinctions. For cluster 11, a major component of the discrepancy is the fraction of hot dust contributing to the K-band: Alonso-Herrero et al. (2004) adopt a fraction ranging from $0 \%$ to $70 \%$, but we derive a fraction closer to $90 \%$ from our best fit (see Section 6.3). The differences in age and dust column density and geometry account for the remaining portion of the discrepancy. Even with these discrepancies, the masses we derive for both clusters and those of Alonso-Herrero et al. (2004) agree to better than $60 \%$, when using their more conservative estimate for cluster 11 .

The area surrounding clusters 5 and 11 hosts a half-dozen Wolf-Rayet stars, mainly of the younger WN type. This would suggest presence of stars/clusters in the narrow age range 2.5-3.5 Myr (Schaerer et al. 1997; Monreal-Ibero et al. 2010; Westmoquette et al. 2013). This is not necessarily in contradiction with the $\sim 1 \mathrm{Myr}$ age we derive for clusters 5 and 11 . The relatively low spatial resolution of ground-based spectroscopy, $\sim 1 "$ " $5-2^{\prime \prime}$ (20-30 pc, or about the size of the panels in Figure 2; see a summary description in Westmoquette et al. 2013), has not enabled accurate location of the stars or clusters responsible for the Wolf-Rayet emission features. This suggests two possible scenarios. In one scenario, the WN stars co-exist in the region with clusters 5 and 11 , and these clusters are the most recent "products" of ongoing star formation over the past few Myr. In the second scenario, the WN features may originate from VMSs (Crowther et al. 2010), hosted in the extremely young clusters 5 and 11 .

Whitmore et al. (2011) suggest a method based on the morphology of the $\mathrm{H} \alpha$ emission in order to classify star clusters according to their ages: the gas morphology is compact and coincident with the star cluster for ages $<$ a few Myr; has a small ring-like structure in clusters up to $\sim 5 \mathrm{Myr}$ of age; has a large, well-formed ring surrounding the cluster for ages in the range 5-10 Myr; and is virtually absent in clusters older than 10 Myr. Whitmore et al. (2014a) adds earlier stages to this classification by including proto-cluster phases based on $\mathrm{CO}$ appearance. When evaluated according to the criteria of these papers, our clusters form a well defined sequence, with a close agreement between our SED-fitting ages and the morphological ages, and with clearly identifiable stages from 2 for cluster 11 (embedded cluster) to 5 for clusters 6-to-10 (intermediate/old cluster).

\subsection{Global Properties of the Clusters}

The total $\mathrm{H} \alpha$ luminosity of NGC5253, corrected for foreground Milky Way extinction and [N $\mathrm{NI}$ contribution, but uncorrected for internal dust attenuation, is $L(\mathrm{H} \alpha)_{\text {total }}=$ $2.2 \times 10^{40} \mathrm{erg} \mathrm{s}^{-1}$ (Kennicutt et al. 2008). About $15 \%$ of the $\mathrm{H} \alpha$ is associated with shock-ionization (Hong et al. 2013), implying that the photo ionized $\mathrm{H} \alpha$ luminosity is $L(\mathrm{H} \alpha)_{\text {phot }}=$ $1.9 \times 10^{40} \mathrm{erg} \mathrm{s}^{-1}$. The sum of the observed $\mathrm{H} \alpha$ luminosity from the star clusters analyzed in this work, also uncorrected for internal dust attenuation, is $L(\mathrm{H} \alpha)_{\text {clusters }}=1.43 \times$ $10^{39} \mathrm{erg} \mathrm{s}^{-1}$ (from Table 3), or about $7.5 \%$ of the total $\mathrm{H} \alpha$. Thus, the brightest 10 clusters contribute almost $10 \%$ of the total (observed) $\mathrm{H} \alpha$ luminosity in this galaxy; indeed, although there are almost 150 young star clusters in the central region of NGC 5253, the vast majority tend to have low mass, i.e., $\lesssim 10^{4} M_{\odot}$ (de Grijs et al. 2013).

A similar argument can be made for the FUV luminosity. The total luminosity density contained within the ACS/SBC 
frame at $\sim 1500 \AA$, corrected for foreground Milky Way extinction, but uncorrected for internal dust attenuation, is $L(1500 \AA) \sim 5.8 \times 10^{38} \mathrm{erg} \mathrm{s}^{-1} \AA^{-1}$. The GALEX FUV luminosity density of NGC 5253 is $L(1500 \AA)_{\text {total }} \sim$ $6.6 \times 10^{38} \mathrm{erg} \mathrm{s}^{-1} \AA^{-1}$, i.e., only about $15 \%$ larger than the amount contained in the HST image. We assume this $15 \%$ discrepancy to be the upper limit to the amount of FUV light outside of the ACS/SBC frame, since both the SBC/F125LP filter and the GALEX/FUV filter have pivot wavelengths at $\sim 1,500 \AA$. The total contribution from the 11 clusters investigated here is $L(1500 \AA)_{\text {clusters }} \sim 2.7 \times 10^{37} \mathrm{erg} \mathrm{s}^{-1} \AA^{-1}$, or about $4 \%-5 \%$ of the total. Star clusters contribute about $20 \%$ of the total observed UV light in nearby star-forming and starburst galaxies (Meurer et al. 1995; Maoz et al. 1996). Thus, the 11 bright clusters represents about $20 \%-25 \%$ of this contribution.

The patchy extinction in the center of NGC 5253 makes it difficult to convert the above numbers to intrinsic luminosities. We adopt a hybrid approach, by combining the ionizing photon flux measured from the free-free emission within the radio nebula (Meier et al. 2002; Turner \& Beck 2004) with the virtually extinction-free $\mathrm{H} \alpha$ emission outside the radio nebula (Calzetti et al. 2004). The two combined yield a total, attenuation-corrected $\mathrm{H} \alpha$ luminosity $L(\mathrm{H} \alpha)_{\text {phot,corrected }} \sim$ $7.1-8.2 \times 10^{40} \mathrm{erg} \mathrm{s}^{-1}$ for the photo-ionized component, about $20 \%-23 \%$ of which is from outside the radio nebula. The attenuation-corrected $\mathrm{H} \alpha$ luminosity is, thus, $\approx 3.5-4$ times larger than the observed one for NGC 5253. The SEDpredicted $\mathrm{H} \alpha$ luminosity for all the 11 clusters is $L(\mathrm{H} \alpha)_{\text {clusters,corrected }} \sim 2.6 \times 10^{40} \mathrm{erg} \mathrm{s}^{-1}$ (Table 6), to which clusters 5 and 11 contribute $22 \%$ and $76 \%$, respectively, and the remaining 9 clusters only contribute a total of $2 \%$. These 9 clusters provide $\sim 3 \%$ of the ionizing photon flux outside the radio nebula; the remaining flux is provided by the smaller clusters and UV-bright diffuse stars that populate the region (Hong et al. 2013). Within the radio nebula, clusters 5 and 11 supply about $40 \%-50 \%$ of the detected ionizing photon flux, i.e., they come short of providing the full ionizing flux by a factor $\sim 2$. We further discuss this discrepancy in Section 6.4.

If unimpeded by dust, cluster 11 would have an absolute magnitude $M_{V}(\mathrm{Vega}) \sim-12.8$, which is about 2-3 $\sigma$ above the mean of the $M_{\text {brightest }}$-SFR relation for star clusters, for the SFR value of NGC 5253 (Whitmore et al. 2014b); this is in the bright envelope, but not outside the range of statistically possible values. Indeed, the dust-free absolute $V$ magnitude of cluster 11 is consistent with the results of Billett et al. (2002), who find that dwarf galaxies tend to host massive clusters more frequently than do their more massive spiral counterparts. Billett et al. (2002) normalize all star clusters to a fiducial age of $10 \mathrm{Myr}$; if cluster 11 is "aged" to $10 \mathrm{Myr}$, its dust-free absolute magnitude would be $M_{V} \sim-12.6$.

\subsection{The Near Infrared Excess of Cluster 11}

The heavily dust-attenuated cluster 11 is anomalously bright in the $\mathrm{J}$ and $\mathrm{H}$ bands, about a factor 2-2.5 than what predicted from the best fit stellar population models. There are four possible causes for this, which we will analyze in turn: (1) excess nebular emission relative to our default model assumption; (2) red supergiants; (3) Pre-main-sequence stars; and (4) hot dust emission. Option 4 has been already considered by other authors for this region, and previously seen in other embedded clusters (Johnson et al. 2004), but will be re-analyzed here in light of our model for the stellar and dust mixture of this cluster.

For option 1, we re-run our best-fit programs using models that implement nebular continuum and line emission with $100 \%$ covering factor, to mimic a tightly confined $\mathrm{H}$ II region around a star cluster. In all cases, we find that the best fit solution has a reduced $\chi^{2}$ that is at least twice as worse as the cases with $50 \%$ covering factor. The reason is because the nebular continuum and line emission contribute to the optical bands as well as the infrared ones, thus requiring a higher degree of dust attenuation to produce the observed SED shape. The higher attenuation pushes the UV model further away from the data, while not compensating enough for the high value of the near-IR data, and ultimately resulting in a poorer fit. Thus, a $50 \%$ covering factor is in better agreement with the data even in this more extreme case, and is in agreement with our measurement procedure for the stellar continuum and emission lines (in which we have applied larger values of the aperture corrections to the lines than to the continuum).

The presence of red supergiants (option 2) is attractive because these contribute significantly in the $\mathrm{J}$ and $\mathrm{H}$ bands. However, cluster 11 is too young to include red supergiants, which would need to be originating from another cluster/ location, along the line of sight of cluster 11 . These red supergiants would also need to be associated with a star cluster that is otherwise heavily obscured at wavelengths shorter than $\mathbf{J}$ and does not emit ionizing photons, since the entire $\mathrm{P} \beta$ and $\mathrm{P} \alpha$ luminosities are fully accounted for by cluster 11 . The $100 \mathrm{Myr}$ old population in the two-populations solution discussed in Section 5.2 is too old to still contain red supergiant stars. In summary, we disfavor this scenario, although we cannot exclude it completely.

Pre-main-sequence stars (option 3) are likely to be present in a young cluster, and to provide a significant contribution to the infrared emission. However, the effect corresponds to an increase of $\sim 30 \%$ in the $\mathrm{K}-$ to- $\mathrm{V}$ luminosity ratio relative to a population without Pre-main-sequence stars (Zackrisson et al. 2001); this is much smaller than that the order-ofmagnitude increase observed (see below).

Option 4, the presence of hot dust surrounding the $\mathrm{P} \alpha$ peak, has been studied already by previous authors, including Vanzi \& Sauvage (2004) and Alonso-Herrero et al. (2004). Vanzi \& Sauvage (2004) used Adaptive-optics near-infrared observations at $\mathrm{Ks}(2.16 \mu \mathrm{m})$ and $\mathrm{L}^{\prime}(3.78 \mu \mathrm{m})$ to determine and measure a peak of emission in these two bands in correspondence of the location of Clusters 5 and 11. The observations have FWHM $=0$ ". 4 , which is comparable to the separation between the two clusters. Vanzi \& Sauvage (2004) modeled the SED of cluster 5 at optical wavelengths combined with their and other measurements at longer wavelengths, employing a physically based model for the dust emission. Vanzi \& Sauvage (2004), however, did not have measurements at wavelengths between I and Ks, and were not aware of the presence of Cluster 11, so the accuracy of their results is difficult to assess. Since cluster 5 does not show any evidence for near-infrared excess based on our SED modeling, we assign those authors' Ks and L' emission to cluster 11.

Alonso-Herrero et al. (2004) measured the emission from the entire central region, targeting an area of about 23-24 pc $(1$. $5 \times 1$." 6 , larger at wavelengths longer than $5 \mu \mathrm{m})$, including both Clusters 5 and 11. They also used a physically based model to account for both the stellar and dust emission of this 


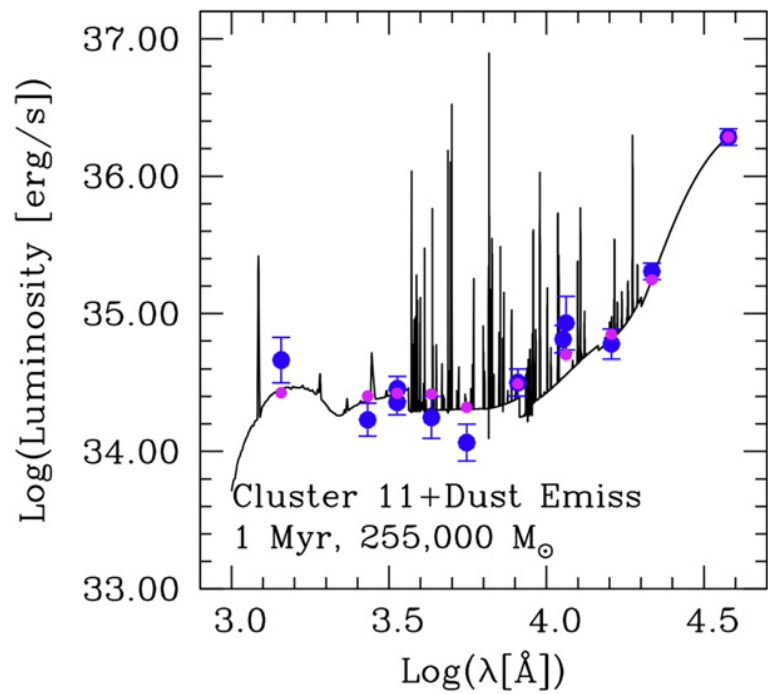

Figure 10. Best fit SEDs for Cluster 11, including stellar, nebular, and dust emission, plus dust attenuation for the stellar and nebular components. The best fit for the stellar, nebular, and dust attenuation components are the same as in Figure 9, while the dust emission is the combination of two modified blackbodies, with temperatures $T_{d, 1} \sim 1100 \mathrm{~K}$ and $T_{d, 2} \sim 440 \mathrm{k}$, and emissivity $\epsilon=1.8$. The two data points longward of $1.6 \mu \mathrm{m}$ are from Vanzi \& Sauvage (2004). Virtually all of the excess in the $\mathrm{J}$ and $\mathrm{H}$ bands can be accounted for with this simple dust emission model.

region, but their model had difficulties in accounting for the emission below the $\mathrm{H}$ band. They ascribed this difficulty to the complexity of the stellar and dust geometry in the region.

Since we are interested mainly in explaining the excess emission at $\mathbf{J}$ and $\mathrm{H}$ for cluster 11 , we use the measurements in the Ks and L' bands from Vanzi \& Sauvage (2004, their Table 3), which are obtained with a smaller aperture size than those of Alonso-Herrero et al. (2004). We attempt a simple modeling of the emission of the dust surrounding this cluster. For the same reason, we do not include measurements at longer wavelengths, because the lower resolution requires larger apertures, and more contamination from the region surrounding cluster 11 . Using our distance for NGC 5253, we derive $L(2.16 \mu \mathrm{m})=2.0 \times 10^{35} \mathrm{erg} \mathrm{s}^{-1} \AA^{-1}$ and $L$ $(3.78 \mu \mathrm{m})=1.9 \times 10^{36} \mathrm{erg} \mathrm{s}^{-1} \AA^{-1}$. An extrapolation of our best-fit stellar population (plus dust attenuation) model shows that the stellar contribution to the $2.16 \mu \mathrm{m}$ emission is about $10 \%$, and is insignificant in the longer wavelength band.

Adding a two-component dust emission model to the stellar + dust attenuation model reproduces the data from $\sim 1500 \AA$ to $\sim 4 \mu \mathrm{m}$ reasonably well, as shown in Figure 10. The twocomponent dust model consists of two modified blackbodies with temperatures $T_{d, 1} \sim 1100 \mathrm{~K}$ and $T_{d, 2} \sim 440 \mathrm{k}$, and emissivity $\epsilon=1.8$. The dust emission model parameters are not the result of a rigorous fitting procedure, thus the derived temperatures are approximate. Furthermore, an emissivity with power law of 1.8 is a gross approximation below $20 \mu \mathrm{m}$ (Draine 2003), but changing the dust emissivity has a small effect on the derived temperatures, for our restricted wavelength range. Our two values for the dust temperature, 1100 and $440 \mathrm{~K}$, bracket the value of $T=570 \mathrm{~K}$ derived by Vanzi \& Sauvage (2004) for the inner shell of their cocoon dust model. Additionally, we have measurements in both the $\mathrm{J}$ and $\mathrm{H}$ bands, which further enable us to place constraints on the dust emission in this range and derive a higher temperature of $1100 \mathrm{~K}$.
There is still some unaccounted for excess in the J-band, although we cannot assess its magnitude, given the size of our uncertainties. Thus, emission from hot dust surrounding cluster 11 appears to be capable of explaining most of the observed excess in the J-band and beyond for the SED of this star cluster. A more physically based model for the dust emission would be required to infer additional properties for the dust, but this is beyond the scope of the present work.

We infer the dust mass associated with these two temperature components, as a sanity check to the reasonability of our dust SED fit. This mass will be a lower limit to the actual dust mass associated with those components, since we are in the optically thick regime: the dust optical depth along the direction of cluster 11 is $\tau_{d}(4 \mu \mathrm{m}) \gtrsim 1.2$, which we derive using the gas column density discussed in the next section and the dust emissivity value at $\sim 4 \mu \mathrm{m}$ from Draine (2004); we use the LMC dust emissivity curve from this paper, on account of the low metallicity in NGC 5253. We derive $M_{\text {dust }}(4 \mu \mathrm{m}) \gtrsim 10 M_{\odot}$, more than $99 \%$ of which is contributed by the cooler of the two dust components, $T_{d, 2} \sim 440 \mathrm{~K}$. This mass is a tiny fraction of the total dust mass in this region, $\sim 10^{5} M_{\odot}$, as derived by probing the cooler dust SED with a peak around 30-40 $\mu \mathrm{m}$ (Vanzi \& Sauvage 2004). It is, however, consistent with the the expectation that only the dust located in close proximity of the massive stars will be heated to high temperatures. For reference, the amount of hot dust surrounding Ultracompact $\mathrm{H}$ II regions in the Milky Way is calculated to be around a few to a few tens of $M_{\odot}$ (Walsh et al. 1999), consistent with our lower limit for the mass in hot dust associated with cluster 11.

The presence of dust as hot as $T_{d} \approx 1100 \mathrm{~K}$ in proximity of cluster 11 supports our SED fitting approach of using a mixed geometry for the stars and dust. In this type of geometry, the dust is likely located close to the UV-emitting stars needed to heat it to high temperatures. Albeit large, the value of the dust temperature is still below the dust sublimation temperature of $\approx 2000 \mathrm{~K}$ (Kobayashi et al. 2011), and is not dissimilar from that found for Ultracompact $\mathrm{H}_{\text {II }}$ regions in the Milky Way, where single massive stars can heat a shell of dust in the natal cloud up to $\sim 1500-1800 \mathrm{~K}$ (Walsh et al. 1999).

\subsection{Energy Balance within the Radio Nebula}

Clusters 5 and 11 are the most prominent ones in the the radio nebula. These clusters have strong hydrogen emission line intensity and concentrated free-free emission (AlonsoHerrero et al. 2004; Turner \& Beck 2004), although several other fainter cluster or massive star candidates are visible in the immediate area $(\leqslant 0$." $6 \sim 9$ pc from cluster 5$)$.

The two clusters share a very young age, $\sim 1 \mathrm{Myr}$, and a common shell of foreground dust, with optical depth $A_{V} \sim$ $1.9 \mathrm{mag}$. However, they also show significant differences. Cluster 5 is about 3.5 times less massive than cluster 11, and is not as deeply buried in dust. Its location in a less dustenshrouded environment than cluster 11 accounts for its prominence as the $\mathrm{H} \alpha$ peak emitter in the galaxy, and for the possibility that $25 \%-50 \%$ of its ionizing photons may be leaking out of the surrounding $\mathrm{H}$ II region (Section 5.2).

Cluster 11 , with a best-fit mass of $2.5 \times 10^{5} M_{\odot}$, is the behemoth in this dwarf galaxy, although not as extreme as inferred in earlier estimates, where values as large as $\sim 10^{6} M_{\odot}$ have been suggested. While more massive than cluster 5, cluster 11 is significantly fainter because it is immersed in a dust cloud with front-to-back optical depth $A_{V} \sim 49 \mathrm{mag}$. 
Clusters 5 and 11 contribute about $50 \%$ of the ionization in the radio nebula and about $35 \%$ of the total in the galaxy, with most of it coming from cluster 11. While they provide a significant fraction of the ionizing photons in both the radio nebula and in NGC 5253, they do not provide the totality. This poses a potential issue of energy balance, which we discuss in the context of the radio nebula. Within this $\sim 20 \mathrm{pc}$ region (Figure 2), clusters 5 and 11 account for $50 \%$ of the free-free emission, for almost the totality of the observed $\mathrm{H} \alpha$ emission, but only for $23 \%$ of the observed $\mathrm{P} \alpha$ emission. The rest of the $\mathrm{P} \alpha$ emission is spread throughout the region of the radio nebula and has a diffuse morphology, as already observed by AlonsoHerrero et al. (2004).

If we double the mass of cluster 11 in order to compensate for the factor $\sim 2$ discrepancy in the free-free emission, the observed stellar continuum photometry is reproduced with a front-to-back optical depth $A_{V} \sim 100$ mag for the dust cloud in which the star cluster is immersed. The $\mathrm{H} \alpha$ emission requires the same optical depth, but the $\mathrm{P} \alpha$ emission only needs $A_{V} \sim$ $20 \mathrm{mag}$, in order to recover the measured luminosity. The discrepancy in the dust optical depth of the two emission lines suggests that this is not a viable scenario. Self-consistent solutions for the available data using a $\sim 5 \times 10^{5} M_{\odot}$ star cluster can only be obtained by modeling independently the stellar continuum and the ionized emission, as discussed in Section 5.2. We disfavor such solutions, on the basis that the lines and continuum of cluster 11 appear spatially correlated.

Our original solution, with a cluster mass of $2.5 \times 10^{5} M_{\odot}$ and front-to-back $A_{V} \sim 50 \mathrm{mag}$, can close the gap between predicted and measured ionizing photon flux in the radio nebula, if young stellar populations produce more ionizing photons than what accounted for by the models we use. Models that include VMSs (Crowther et al. 2010; Doran et al. 2013) have the potential to increase the ionizing photon flux by up to a factor of 2 , with smaller impact on the stellar continuum luminosities. The amount of diffuse $\mathrm{P} \alpha$ in the radio nebula suggests that about half of the ionizing photons leak out of the clusters into the region. This is similar to the fraction of ionizing photons leakage found by Johson et al. (2009) for the compact, dusty clusters in the starburst galaxy SBS 0335-052. The observed $\mathrm{H} \alpha / \mathrm{P} \alpha /$ free-free intensity ratios outside the two clusters can be fully explained by foreground dust with color excess $E(B-V) \sim 2 \mathrm{mag}$, a factor almost three lower than the total extinction in cluster 11 . The gas outflow present in the area, as inferred from the broad component in the $\mathrm{H} \alpha$ emission (Monreal-Ibero et al. 2010; Westmoquette et al. 2013), may create favorable conditions for leakage of ionizing photons, by causing the ISM to become porous.

The diffuse nature of the $\mathrm{P} \alpha$ emission outside of clusters 5 and 11 further suggests that this emission may originate from other stars and/or clusters in the region, some of which may be themselves buried in dust and undetected except at wavelengths longer than a few micron.

\subsection{The Environment of the Radio Nebula}

As already indicated by a number of previous studies (see Introduction), the radio nebula is the youngest and most active area of star formation in NGC 5253. It is also the region of intersection between the central starburst and the dust lane, which accounts for its significant dust content.

The dust cloud enshrouding cluster 11 may appear exceptional, with its $A_{V}=49 \mathrm{mag}$, but it is comparable to clouds in other galaxies, including those in the Milky Way (see, e.g., the Ultracompact $\mathrm{H}_{\text {II }}$ region $\mathrm{W} 3(\mathrm{OH})$, Turner \& Welch 1984). Adopting the total hydrogen column density-to-color excess relation of Bohlin et al. (1978), rescaled to the lower metallicity of NGC 5253, the optical depth of the cloud corresponds to a hydrogen column density $N(\mathrm{H}) \sim$ $2 \times 10^{23} \mathrm{~cm}^{-2}$. Although large, this value is comparable to that observed in some of the massive dense clouds toward the center of the Milky Way (e.g., Kauffmann et al. 2013). If distributed uniformly throughout a region of about $1 \mathrm{pc}$ size, the observed gas column density would correspond to a dust density $\rho_{d} \sim 7 \times 10^{-22} \mathrm{~g} \mathrm{~cm}^{-3}$, only a factor of a few lower than the dust densities typical of Ultracompact $\mathrm{H}$ II regions in the Milky Way (Walsh et al. 1999). As an independent check, the $\mathrm{H}_{2}$ density calculated by Turner et al. (2015) corresponds to $\rho_{\text {gas }} \sim 1.56 \times 10^{-19} \mathrm{~g} \mathrm{~cm}^{-3}$, or a dust/gas ratio $\sim 0.0045$ for the cloud of cluster 11, which is only slightly lower than the values measured for nearby galaxies (Draine et al. 2007). This ratio could be lower still, since the $\mathrm{H}_{2}$ density is derived from the $\mathrm{CO}(3-2)$ transition, which yields a lower limit to the actual molecular gas density. High-resolution HI maps of the region only show HI absorption, which sets a lower limit of $5 \times 10^{20} \mathrm{~cm}^{-2}$ to the HI column density (Kobulnicky \& Skillman 1995).

The virial mass contained in a region about $43 \times 23 \mathrm{pc}$, roughly the size of the region displayed in either panel of Figure 2, is about 1.2-1.3 $\times 10^{6} M_{\odot}$ (Turner et al. 2015, scaled to our distance). If clusters 5 and 11 provide most of the stellar mass in the region, the star formation efficiency is SFE $0.25-0.30$, about a factor 2 lower than the estimate ${ }^{48}$ of Turner et al. (2015). The SFE we derive is comparable to the SFE of clusters in our own Milky Way, but larger than the SFE 5\%$10 \%$ measured when including the entire molecular cloud (Lada \& Lada 2003). Whether or not clusters 5 and 11 will remain bound depends on both the value of the local (clustersize) SFE and the ratio of the gas removal to the crossing timescales (Parmentier et al. 2009).

If they remain bound and do not lose significant mass, clusters 5 and 11 are massive enough that they could be progenitors of globular clusters. Globular clusters, like those in the halo of our own Milky Way and other galaxies, have masses in the range $\approx 10^{4}-10^{6} M_{\odot}$ (Fall et al. 2009). The mass loss of an evolving cluster depends on a number of factors, including the nature/origin of the second population (Schaerer \& Charbonnel 2011), but under most scenarios, both clusters 5 and 11 would retain sufficient mass to remain within the range of globular clusters. Clusters 5 and 11 are two of the youngest among the very massive clusters (super-star-clusters) detected in nearby galaxies, such as those in He2-10 (Kobulnicky \& Johnson 1999), NGC 1569 (Hunter et al. 2000), M82 (Smith et al. 2006), SBS 0335-052 (Johson et al. 2009), the Antennae (Whitmore et al. 2010), and NGC 1705 (Martins et al. 2012), to name a few. As such, they provide important case studies to test theories of multiple populations in globular clusters (e.g., de Mink et al. 2009; Bastian et al. 2015).

\footnotetext{
$\overline{48}$ The star formation efficiency decreases to $\mathrm{SFE} \sim 0.15$, if we adopt a topheavy stellar IMF with a lower cut-off of $1 M_{\odot}$; this cut-off is lower than the $3 M_{\odot}$ proposed by Turner et al. (2015).
} 


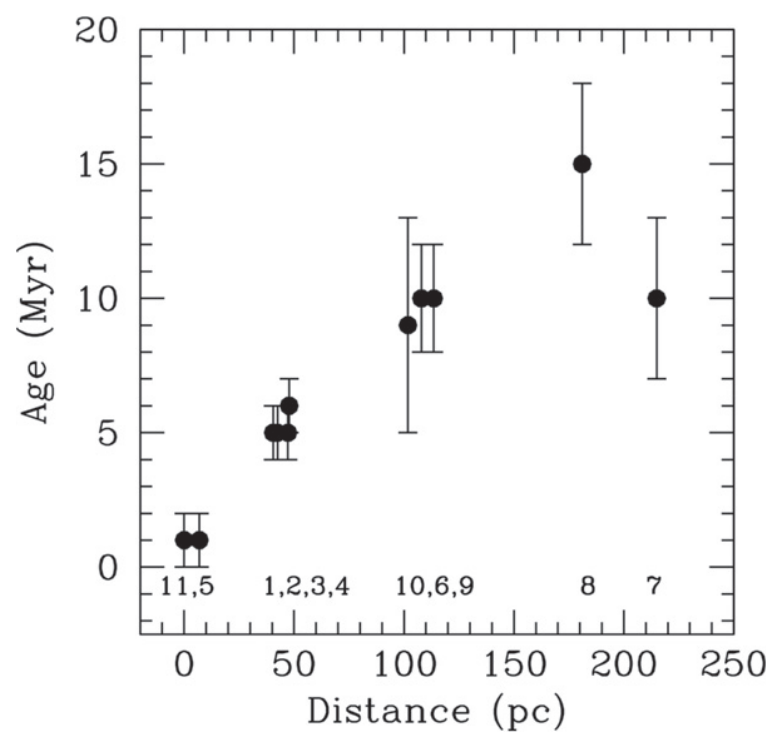

Figure 11. Ages, with their $1 \sigma$ uncertainty, of the 11 star clusters as a function of their distance from cluster 11. For each data point, the corresponding cluster number is indicated at the bottom of the figure. A systematic trend is observed, with the older clusters located further away from the location of cluster 11, i.e., from the region of most active star formation in the galaxy.

\subsection{The Starburst in NGC 5253}

The youth of the two clusters in the radio nebula has an interesting implication for the estimate of the SFR in this galaxy from its TIR luminosity, $L_{\mathrm{TIR}}=3.7 \times 10^{42} \mathrm{erg} \mathrm{s}^{-1}$. The majority of the TIR emission in NGC 5253 originates from the same region, as suggested by the point-like emission in the Spitzer images (Dale et al. 2009) and by mid-IR imaging (Gorjian et al. 2001). If we use a scaling factor between $L$ (TIR) and SFR appropriate for a population as young as $2 \mathrm{Myr}$ (Calzetti 2013), we obtain SFR(TIR) $=0.31 M_{\odot} \mathrm{yr}^{-1}$, a factor almost 3 larger than what would be inferred using the standard calibration. The higher SFR(TIR) is consistent with the range of SFRs derived from the free-free emission, SFR $\sim 0.3-0.36$ $M_{\odot} \mathrm{yr}^{-1}$, suggesting that, within the uncertainties, we see no obvious indication for significant direct absorption of ionizing photons by dust. Adding the UV, which originates in separate regions from the IR, we get a total SFR for NGC 5253 $\mathrm{SFR}=0.37 M_{\odot} \mathrm{yr}^{-1}$, when using a timescale of $10 \mathrm{Myr}$ for the SFR(UV), which is appropriate for the youth of the UVemitting area.

Moving away from the central radio nebula, the clusters increase in age for increasing distance from clusters 5 and 11 (Figures 1 and 11). Clusters $1-4$ have best-fit ages around 5-6 Myr, while clusters 7-10 are consistent with ages 9-15 Myr. The "association" of clusters 1-4, all with comparable ages and spread over a small area of $\sim 170 \mathrm{pc}^{2}$, agrees with the micro-level hierarchy picture discussed by Efremov \& Elmegreen (1998); these authors find that in the Magellanic Clouds small regions form stars over a short period of time. UV-bright star clusters to the north of the radio nebula have ages in the range 4-8 Myr (Tremonti et al. 2001). de Grijs et al. (2013) also recovers typically young ages for the star clusters in the central region of NGC 5253, between a few $10^{6}$ years and a few $10^{7}$ years. This suggests a progressive concentration of the most recent event of star formation from a larger, $\sim 300 \mathrm{pc}$, region to the smaller area, $\sim 20 \mathrm{pc}$, of the radio nebula. Covering $300 \mathrm{pc}$ in $\sim 15 \mathrm{Myr}$ requires a propagation velocity of the perturbation of about $20 \mathrm{~km} \mathrm{~s}^{-1}$. This is larger than the typical sound speed of a few $\mathrm{km} \mathrm{s}^{-1}$ of the ISM, but is consistent with the velocities of a few tens of $\mathrm{km} \mathrm{s}^{-1}$ expected for the propagation of turbulence in a multi-phase ISM (e.g., Bournaud et al. 2011). External triggers, such as the recent-past interaction with M83 and/or gas infall, are required for such large-scale propagating disturbances, although internal triggers (e.g., shocks from earlier events that produce sequential star formation, see, Whitmore et al. 2010) may also play a role. The region within the central $\approx 300$ pc of NGC 5253 is likely to have been the stage of one continuous and connected event of star formation over at least the past $\sim 15 \mathrm{Myr}$. This is in agreement with other estimates that indicate relatively constant levels of SFR over the past few hundred Myr (McQuinn et al. 2010; Harbeck et al. 2012), although these studies cannot discriminate between continuous or sporadic star formation over this timescale.

The energetics that drive the ISM kinematics in NGC 5253 are also consistent with a continuous star formation event over at least this timescale. The measured $\mathrm{H} \alpha$ luminosity of the shocked gas, $L(\mathrm{H} \alpha)_{\text {shock }} \sim 3 \times 10^{39} \mathrm{erg} \mathrm{s}^{-1}$ (Hong et al. 2013, rescaled to our distance), corresponds to a mechanical luminosity of $L_{\text {mech }} \sim 7 \times 10^{40} \mathrm{erg} \mathrm{s}^{-1}$ (Rich et al. 2010), for radiative shocks and the shock velocity measured by Marlowe et al. (1995). For constant star formation and our adopted Kroupa IMF, the mechanical energy requirements can be satisfied with $\mathrm{SFR} \sim 0.2 M_{\odot} \mathrm{yr}^{-1}$ over the past $10-15 \mathrm{Myr}$. The five star clusters in this age range, 6-to-10, correspond to a cumulative mass of $\sim 1.6 \times 10^{5} M_{\odot}$ (about $1 / 2$ of the sum of the masses of 5 and 11), and may have contributed, in the past, about $1 / 3$ of the mechanical energy needed to support the shocks. The rest of the energy requirement has come from the remaining young star clusters and diffuse stars in the region. When they will have aged enough to start producing supernova explosions, clusters 5 and 11 will be able to supply about $2 / 3$ of the mechanical energy requirement of the present-day shocks.

\section{SUMMARY AND CONCLUSIONS}

The combination of new and archival HST imaging data of the central $300 \mathrm{pc}$ in the starburst galaxy NGC 5253 has enabled us to derive, with unprecedented accuracy, ages, masses, and extinctions for the brightest among the star clusters in the starburst. The HST data cover the full wavelength range from $\sim 1500 \AA$ to $1.9 \mu \mathrm{m}$ in 10 continuum bands and 3 narrow bands, which include the emission lines of $\mathrm{H} \alpha, \mathrm{P} \beta$, and $\mathrm{P} \alpha$. The 11 star clusters analyzed here include the two young clusters located within the central dusty radio nebula, in addition to nine clusters distributed across the UV-bright starburst.

The multi-wavelength SED of each cluster has been fit with models that include stellar populations, gas emission, and dust attenuation (and, for cluster 11, dust emission). Models that include only foreground dust are sufficient to produce excellent fits for the 10 bright clusters, while the heavily dust extincted cluster 11 requires a geometry that includes a combination of foreground and mixed dust.

Because of its location within a region of high optical depth, this is the first time the SED of cluster 11 has been fit shortward of the J-band. This is also the first time extensive stellar continuum photometry, from the UV to the near-IR, is available 
for a natal cluster. Albeit counter-intuitive, cluster 11 is detectable in all optical and UV bands, mainly because dust mixed with stars dims the light more readily than reddening it (Calzetti 2001). Like for all other clusters analyzed in this paper, the SED of cluster 11 can be fit with a standard Kroupa (2001) IMF in the mass range $0.1-120 M_{\odot}$, and does not require truncations or other modifications. The resulting best-fit age, mass, and total dust attenuation of cluster 11 yield a selfconsistent picture of this source, also in agreement with independent constraints.

All clusters studied here are younger than $\sim 15 \mathrm{Myr}$, in agreement with previous results, with a systematic trend for the age to become younger when moving from the outskirts of the starburst toward its center, where the radio nebula is located. The picture that emerges is for the current burst of star formation to have been a continuous event over the past $\sim 15 \mathrm{Myr}$, and to have concentrated inward, forming a hierarchical "age" structure similar to those discussed by Efremov \& Elmegreen (1998) for the LMC. Dwarf starburst galaxies, including NGC 5253, do tend, in fact, to display a single dominant region of connected star formation hierarchical in structure (Elmegreen et al. 2014). SFR indicators calibrated for the youth of the starburst yield SFR $\sim 0.4 \odot \mathrm{yr}^{-1}$ for the galaxy, or about three times higher than using standard SFR calibrations.

The nine star clusters located outside the radio nebula have masses in the range $0.5-5 \times 10^{4} M_{\odot}$, and dust attenuations $A_{V}$ $\lesssim 1$ mag. The relatively low $A_{V}$ value is consistent with the location of the clusters in the UV-bright region of the starburst. Other indicators show dust attenuation to be low in this region in general. The nine clusters provide today a minimal contribution, less than $2 \%$, to the total ionizing photons in the galaxy.

The two most massive clusters in our sample, clusters 5 and 11 , are both located inside the dusty radio nebula. Their large masses, $7.5 \times 10^{4} M_{\odot}$ and $2.5 \times 10^{5} M_{\odot}$, respectively, are consistent with having been born in a high-density environment. Both clusters are extremely young, with best fit ages $1 \pm$ 1 Myr. Neither has reached a stage when supernovae are starting to affect the local environment, including blowing out the natal dust cloud, which accounts for their significant dust attenuation. Energy balance between the ionizing photon rate recovered from the free-free emission and the total far-infrared luminosity suggests that, albeit buried in a significant dust cloud, the clusters in the radio nebula do not suffer from significant direct absorption of ionizing photons by dust.

Both clusters are behind a dust layer of $A_{V}<2 \mathrm{mag}$, which is likely the outer dust shell of the radio nebula. Cluster 5 is behind only this dust layer, which is still sufficiently transparent to enable this cluster to be the $\mathrm{H} \alpha$ peak in the galaxy. Cluster 11 is attenuated by an additional dust cloud with front-to-back optical depth $A_{V} \sim 49 \mathrm{mag}$, mixed with its stars. This dust cocoon has many characteristics typical of those observed in Milky Way Ultracompact $\mathrm{H}_{\text {II }}$ regions, including a maximum temperature of $\sim 1100 \mathrm{~K}$ for the dust closest to the massive stars. It is also responsible for about $60 \%, 65 \%$, and $90 \%$ of the emission observed in the $\mathrm{J}, \mathrm{H}$, and $\mathrm{K}$ bands, respectively.

The mass of cluster 11 places it a factor $\approx 2-4$ below the $\sim 0.6-1.2 \times 10^{6} M_{\odot}$ value inferred in previous studies. Except for its "dusty" condition, owed presumably to its youth, this cluster's other characteristics are relatively normal. For instance, it is within $2-3 \sigma$ of the relation between the most massive clusters and SFR in galaxies, and is similar to massive clusters recovered in other dwarf galaxies. Its mass is inbetween those of the scaled OB association NGC 604 in M 33 (Maiz-Apellaniz 2001) and the massive cluster NGC346 in the SMC (Portegies Zwart et al. 2010). For the level of dust attenuation and for its youth, cluster 11 is reminiscent of the super-star-cluster WS 80 in the Antennae galaxy (Whitmore \& Zhang 2002), although it is also about 16 times less massive. Based on the self-consistency of several indicators, we do not believe to be missing a large fraction of the mass of cluster 11 .

Clusters 5 and 11 provide about half of the ionizing photons in the radio nebula, and about one third of the ionizing flux in the entire galaxy. The remaining $50 \%$ of the nebula's ionizing photons are diffuse, suggesting leakage of ionizing photons from the immediate surroundings of the two clusters into the nebula's region. Once they start producing supernovae, clusters 5 and 11 will supply about $2 / 3$ of its mechanical luminosity requirements to support the current level of shocks. Thus, by themselves, the two clusters can sustain a significant fraction of the energy requirements of NGC 5253. The remaining energy needs to be supplied by other sources, which may include: other stars and star clusters within the area of the radio nebula, VMSs (Crowther et al. 2010; Doran et al. 2013), and/or rotating massive stars (Leitherer et al. 2014). The potential presence of VMSs is consistent with the detection of WolfRayet features of WN type in the region of the radio nebula. Follow-up high-spatial-resolution spectroscopy of these clusters has the potential to reveal signatures of rare star formation products and address this open question.

Based on observations made with the NASA/ESA Hubble Space Telescope, obtained at the Space Telescope Science Institute, which is operated by the Association of Universities for Research in Astronomy, Inc., under NASA contract NAS 5-26555. These observations are associated with program \# 13364. Support for program \# 13364 was provided by NASA through a grant from the Space Telescope Science Institute.

Based also on observations made with the NASA/ESA Hubble Space Telescope, and obtained from the Hubble Legacy Archive, which is a collaboration between the Space Telescope Science Institute (STScI/NASA), the Space Telescope European Coordinating Facility (ST-ECF/ESA) and the Canadian Astronomy Data Centre (CADC/NRC/CSA).

This research has made use of the NASA/IPAC Extragalactic Database (NED) which is operated by the Jet Propulsion Laboratory, California Institute of Technology, under contract with the National Aeronautics and Space Administration.

Part of this work was conducted while D.C. was a Raymond and Beverley Sackler Distinguished Visitor at the Institute of Astronomy, University of Cambridge (UK), and an Overseas Fellow at the Churchill College (Cambridge, UK). D.C. acknowledges the kind hospitality of both the Institute and the College. A.S.E. was supported by the Taiwan, R.O.C. Ministry of Science and Technology grant MoST 102-2119-M001-MY3. M.F. acknowledges support by the Science and Technology Facilities Council [grant number ST/L00075X/1]. D.A.G. kindly acknowledges financial support by the German Research Foundation (DFG) through grant GO 1659/3-2. E.Z. acknowledges research funding from the Swedish Research Council (project 2011-5349). 


\section{REFERENCES}

Alonso-Herrero, A., Takagi, T., Baker, A. J., et al. 2004, ApJ, 612, 222 Anders, P., Kotulla, R., de Grijs, R., \& Wicker, J. 2013, ApJ, 778, 138 Asplund, M., Grevesse, N., Sauval, A. J., \& Scott, P. 2009, ARA\&A, 47, 481 Bastian, N., Cabrera-Ziri, I., \& Salaris, M. 2015, MNRAS, 449, 3333 Beck, S. C., Turner, J. L., Ho, P. T. P., Lacy, J. H., \& Kelly, D. M. 1996, ApJ, 457,610

Billett, O. H., Hunter, D. A., \& Elmegreen, B. G. 2002, AJ, 123, 1454

Bohlin, R. C., Savage, B. D., \& Drake, J. F. 1978, ApJ, 224, 132

Bournaud, F., Chapon, D., Teyssier, R., et al. 2011, ApJ, 730, 4

Bresolin, F. 2011, ApJ, 729, 56

Caldwell, N., \& Phillips, M. M. 1989, ApJ, 338, 789

Calzetti, D. 2001, PASP, 113, 1449

Calzetti, D. 2013, in Secular Evolution of Galaxies, ed. J. Falcón-Barroso \& J. H. Knapen (Cambridge: Cambridge Univ. Press), 419

Calzetti, D., Armus, L., Bohlin, R. C., et al. 2000, ApJ, 533, 682

Calzetti, D., Conselice, C. J., Gallagher, J. S., III, \& Kinney, A. L. 1999, AJ, 118,797

Calzetti, D., Harris, J., Gallagher, J. S., et al. 2004, AJ, 127, 1405

Calzetti, D., Kinney, A. L., \& Storchi-Bergmann, T. 1994, ApJ, 429, 582

Calzetti, D., Lee, J. C., Sabbi, E., et al. 2015, AJ, 149, 51

Calzetti, D., Meurer, G. R., Bohlin, R. C., et al. 1997, AJ, 114, 1834

Campbell, A., Terlevich, R., \& Melnick, J. 1986, MNRAS, 223, 811

Cerviño, M., \& Luridiana, V. 2004, A\&A, 413, 145

Chandar, R., Leitherer, C., Tremonti, C. A., et al. 2005, ApJ, 628, 210

Cook, D. O., Dale, D. A., Johnson, B. D., et al. 2014, MNRAS, 445, 899

Cresci, G., Vanzi, L., \& Sauvage, M. 2005, A\&A, 433, 447

Crowther, P. A., Schnurr, O., Hirschi, R., et al. 2010, MNRAS, 408, 731

Dale, D. A., Cohen, S. A., Johnson, L. C., et al. 2009, ApJ, 703, 517

Davidge, T. J. 2007, AJ, 134, 1799

de Grijs, R., Anders, P., Zackrisson, E., \& Östlin, G. 2013, MNRAS, 431, 2917

de Mink, S. E., Pols, O. R., Langer, N., \& Izzard, R. G. 2009, A\&A, 507, L1

de Mink, S. E., Sana, H., Lange, N., Izzard, R. G., \& Schneider, F. R. N. 2014, ApJ, 782, 7

Doran, E. I., Crowther, P. A., de Koter, A., et al. 2013, A\&A, 558, A134

Draine, B. T. 2003, ARA\&A, 41, 241

Draine, B. T. 2004, in The Cold Universe, Vol. 32, ed. A. W. Blain et al. (Berlin: Springer), 213

Draine, B. T., Dale, D. A., Bendo, G., et al. 2007, ApJ, 663, 866

Efremov, Y. N., \& Elmegreen, B. G. 1998, MNRAS, 299, 588

Eldridge, J. J., \& Stanway, E. R. 2009, MNRAS, 400, 1019

Elmegreen, D. M., Elmegreen, B. G., Adamo, A., et al. 2014, ApJL, 787, L15

Fall, S. M., Chandar, R., \& Whitmore, B. C. 2009, ApJ, 704, 453

Ferland, G. J., Porter, R. L., van Hoof, P. A. M., et al. 2013, RMexAA, 49, 137

Fitzpatrick, E. L. 1999, PASP, 111, 63

Freedman, W. L., Madore, B. F., Gibson, B. K., et al. 2001, ApJ, 553, 47

Gazak, J. Z., Bastian, N., Kudritzki, R.-P., et al. 2013, MNRAS, 430, L35

Girardi, L., Bressan, A., Bertelli, G., \& Chiosi, C. 2000, A\&AS, 141, 371

Gorjian, V., Turner, J. L., \& Beck, S. C. 2001, ApJL, 554, L29

Grimes, J. P., Heckman, T., Aloisi, A., et al. 2009, ApJS, 181, 272

Harbeck, D., Gallagher, J. S., III, \& Crnojevic, D. 2012, MNRAS, 422, 629

Harris, J., Calzetti, D., Gallagher, J. S., Smith, D. A., \& Conselice, C. J. 2004, ApJ, 603, 503

Hong, S., Calzetti, D., Gallagher, J. S. III, et al. 2013, ApJ, 777, 63

Hunter, D. A., O'Connell, R. W., Gallagher, J. S., \& Smecker-Hane, T. A. 2000, AJ, 120, 2383

Johnson, K. E., Indebetouw, R., Watson, C., \& Kobulnicky, H. A. 2004, AJ, 128,610

Johnson, K. E., Vacca, W. D., Leitherer, C., Conti, P. S., \& Lipscy, S. J. 1999, AJ, 117, 1708

Johson, K. E., Hunt, L. K., \& Reines, A. E. 2009, AJ, 137, 3788

Karachentsev, I. D., Tully, R. B., Dolphin, A., et al. 2007, AJ, 133, 504

Kauffmann, J., Pillai, T., \& Zhang, Q. 2013, ApJL, 765, L35

Kennicutt, R. C., \& Evans, N. J. 2012, ARA\&A, 50, 531

Kennicutt, R. C., Lee, J. C., Funes, S. J., Sakai, S., \& Akiyama, S. 2008, ApJS, 178,247

Kobayashi, H., Kimura, H., Watanabe, S., Yamamoto, T., \& Müller, S. 2011, EP\&S, 63, 1067

Kobulnicky, H. A., \& Johnson, K. E. 1999, ApJ, 527, 154

Kobulnicky, H. A., \& Skillman, E. D. 1995, ApJL, 454, L121

Kobulnicky, H. A., Skillman, E. D., Roy, J.-R., Walsh, J. R., \& Rosa, M. R. 1997, ApJ, 277, 679

Koehler, K., Langer, N., de Koter, A., et al. 2015, A\&A, 573, A71

Koekemoer, A. M., McLean, B., McMaster, M., \& Jenkner, H. 2006, The 2005 HST Calibration Workshop: Hubble after the Transition to Two-gyro
Mode, ed. A. M. Koekemoer, P. Goudfrooij \& L. L. Dressel (Washington, D.C.: NASA), 384

Kreckel, K., Groves, B., Schinnerer, E., et al. 2013, ApJ, 771, 62

Kroupa, P. 2001, MNRAS, 322, 231

Lada, C. J., \& Lada, E. A. 2003, ARA\&A, 41, 67

Lee, M. G., Chandar, R., \& Whitmore, B. C. 2005, AJ, 130, 2128

Leitet, E., Bergvall, N., Hayes, M., Linné, S., \& Zackrisson, E. 2013, A\&A, 553, A106

Leitherer, C., Eckström, S., Meynet, G., et al. 2014, ApJS, 212, 14

Leitherer, C., Schaerer, D., Goldader, J. D., et al. 1999, ApJS, 123, 3

Lopez-Sanchez, A. R., Esteban, C., Garcia-Rojas, J., Peimbert, M., \& Rodriguez, M. 2007, ApJ, 656, 168

Lopez-Sanchez, A. R., Koribalski, B. S., van Eymeren, J., et al. 2012, MNRAS, 419, 1051

Maiz-Apellaniz, J. 2001, ApJ, 563, 151

Maoz, D., Barth, A. J., Sternberg, A., et al. 1996, AJ, 111, 2248

Marlowe, A. T., Heckman, T. M., Wyse, R. F. G., \& Schommer, R. 1995, ApJ, 438, 563

Martin, C. L. 1998, ApJ, 506, 222

Martin-Hernandez, N. L., Schaerer, D., \& Sauvage, M. 2005, A\&A, 429, 449

Martins, F., Foerster Schreiber, N. M., Eisenhauer, F., \& Lutz, D. 2012, A\&A, 547, A17

McQuinn, K. B. W., Skillman, E. D., Cannon, J. M., et al. 2010, ApJ, 724, 49

Meier, D. S., Turner, J. L., \& Beck, S. C. 2002, AJ, 124, 877

Meurer, G. R., Heckman, T. M., Leitherer, C., et al. 1995, AJ, 110, 2665

Meynet, G., Schaller, G., Schaerer, D., \& Charbonnel, C. 1994, A\&AS, 103, 97

Monreal-Ibero, A., Vlchez, J. M., Walsh, J. R., \& Muñoz-Tuñon, C. 2010, A\&A, 517, 27

Monreal-Ibero, A., Walsh, J. R., \& Vlchez, J. M. 2012, A\&A, 544, A60

Monreal-Ibero, A., Walsh, J. R., Westmoquette, M. S., \& Vlchez, J. M. 2013, A\&A, 553, A57

Moorwood, A. F. M., \& Glass, I. M. 1982, A\&A, 115, 84

Moustakas, J., \& Kennicutt, R. C. 2006, ApJS, 164, 81

Parmentier, G., Goodwin, S. P., Kroupa, P., \& Baumgardt, H. 2009, ApJ, 678, 347

Pellerin, A., \& Robert, C. 2007, MNRAS, 381, 288

Popescu, B., \& Hanson, M. M. 2010, ApJL, 713, L21

Portegies Zwart, S. F., McMillan, S. L. W., \& Gieles, M. 2010, ARA\&A, 48, 431

Reines, A. E., Nidever, D. L., Whelan, D. G., \& Johnson, K. E. 2010, ApJ, 708, 26

Rich, J. A., Dopita, M. A., Kewley, L. J., \& Rupke, D. S. N. 2010, ApJ, 721,505

Rieke, G. H., Lebofsky, M. J., \& Walker, C. E. 1988, ApJ, 325, 679

Sakai, S., Ferrarese, L., Kennicutt, R. C., \& Saha, A. 2004, ApJ, 608, 42

Sana, H., de Mink, S. E., de Koter, A., et al. 2012, Sci, 337, 444

Schaerer, D., \& Charbonnel, C. 2011, MNRAS, 413, 2297

Schaerer, D., Contini, T., Kunth, D., \& Meynet, G. 1997, ApJL, 481, L75

Schlafly, E. F., \& Finkbeiner, D. P. 2011, ApJ, 737, 103

Schneider, F. R. N., Izzard, R. G., de Mink, S. E., et al. 2014, ApJ, 780, 117

Schneider, F. R. N., Izzard, R. G., Langer, N., \& de Mink, S. E. 2015, ApJ, 805,20

Smith, L. J., Westmoquette, M. S., Gallagher, J. S., et al. 2006, MNRAS, 370,513

Storchi-Bergmann, T., Kinney, A. L., \& Challis, P. 1995, ApJS, 98, 103

Strickland, D. K., \& Stevens, I. R. 1999, MNRAS, 306, 43

Summers, L. K., Stevens, I. R., Strickland, D. K., \& Heckman, T. M. 2004, MNRAS, 351, 1

Thim, F., Tammann, G. A., Saha, A., et al. 2003, ApJ, 590, 256

Tremonti, C. A., Calzetti, D., Leitherer, C., \& Heckman, T. M. 2001, ApJ, 555,322

Turner, J. L., \& Beck, S. C. 2004, ApJL, 602, L85

Turner, J. L., Beck, S. C., Bendford, D. J., et al. 2015, Natur, 519, 331

Turner, J. L., Beck, S. C., \& Ho, P. T. P. 2000, ApJL, 532, L109

Turner, J. L., Beck, S. C., \& Hurt, L. R. 1997, ApJL, 474, L11

Turner, J. L., \& Welch, W. J. 1984, ApJL, 287, L81

van den Bergh, S. 1980, PASP, 92, 122

Vanzi, L., \& Sauvage, M. 2004, A\&A, 415, 509

Vazquez, G. A., \& Leitherer, C. 2005, ApJ, 621, 695

Walsh, A. J., Burton, M. G., Hyland, A. R., \& Robinson, G. 1999, MNRAS, 309,905

Walsh, J. R., \& Roy, J.-R. 1989, MNRAS, 239, 297

Westmoquette, M. S., James, B., Monreal-Ibero, A., \& Walsh, J. R. 2013, A\&A, 550, A88

Whitmore, B. C., Brogan, C., \& Chandar, R. 2014a, ApJ, 795, 156

Whitmore, B. C., Chandar, R., Bowers, A. S., et al. 2014b, AJ, 147, 78 
Whitmore, B. C., Chandar, R., Kim, H., et al. 2011, ApJ, 729, 78

Whitmore, B. C., Chandar, R., Schweizer, F., et al. 2010, AJ, 140, 75

Whitmore, B. C., \& Zhang, Q. 2002, AJ, 124, 1418

Yusof, N., Hirschi, R., Meynet, G., et al. 2013, MNRAS, 433, 1114

Zackrisson, E., Bergvall, N., Oloffson, K., \& Siebert, A. 2001, A\&A, 375, 814
Zackrisson, E., Rydberg, C.-E., Schaerer, D., Östlin, G., \& Tuli, M. 2011, ApJ, 740,13

Zastrow, J., Oey, M. S., Veilleux, S., \& McDonald, M. 2013, ApJ, 779, 76

Zastrow, J., Oey, M. S., Veilleux, S., McDonald, M., \& Martin, C. L. 2011, ApJL, 741, L17 